\title{
Gaugino production in proton-proton collisions at a center-of-mass energy of $8 \mathrm{TeV}$
}

\author{
Benjamin Fuks, ${ }^{a}$ Michael Klasen, ${ }^{b}$ David R. Lamprea ${ }^{b}$ and Marcel Rothering ${ }^{b}$ \\ ${ }^{a}$ Institut Pluridisciplinaire Hubert Curien/Département Recherches Subatomiques, \\ Université de Strasbourg/CNRS-IN2P3, \\ 23 rue du Loess, F-67037 Strasbourg, France \\ ${ }^{b}$ Institut für Theoretische Physik, Westfälische Wilhelms-Universität Münster, \\ Wilhelm-Klemm-Straße 9, D-48149 Münster, Germany \\ E-mail: benjamin.fuks@iphc.cnrs.fr, michael.klasen@uni-muenster.de, \\ david.lamprea@uni-muenster.de, marcel.rothering@uni-muenster.de
}

AbStract: Motivated by hints for a light Standard Model-like Higgs boson and a shift in experimental attention towards electroweak supersymmetry particle production at the CERN LHC, we update in this paper our precision predictions at next-to-leading order of perturbative QCD matched to resummation at the next-to-leading logarithmic accuracy for direct gaugino pair production in proton-proton collisions with a center-of-mass energy of $8 \mathrm{TeV}$. Tables of total cross sections are presented together with the corresponding scale and parton density uncertainties for benchmark points adopted recently by the experimental collaborations, and figures are presented for up-to-date model lines attached to them. Since the experimental analyses are currently obtained with parton showers matched to multi-parton matrix elements, we also analyze the precision of this procedure by comparing invariant-mass and transverse-momentum distributions obtained in this way to those obtained with threshold and transverse-momentum resummation.

Keywords: Supersymmetry Phenomenology, Monte Carlo Simulations

ARXIV EPRINT: 1207.2159 


\section{Contents}

1 Introduction 1

2 Total cross sections at $\sqrt{s}=8 \mathrm{TeV} \quad 3$

2.1 Benchmark points 3

2.2 Total cross sections for the benchmark points 1, 18, and $31 \quad 6$

2.3 Total cross sections for model lines 10.1, 10.3, and $40.1 \quad 9$

3 Comparisons of invariant-mass and transverse-momentum distributions 10

$\begin{array}{ll}3.1 & \text { Distributions in the invariant mass of the gaugino pair } \\ \end{array}$

$\begin{array}{ll}3.2 & \text { Distributions in the transverse momentum of the gaugino pair }\end{array}$

$\begin{array}{lll}4 & \text { Conclusion } & 19\end{array}$

A Total cross sections at $\sqrt{s}=7 \mathrm{TeV}$ for benchmark points 1,18 , and 3121

\section{Introduction}

For many years, the Standard Model (SM) of particle physics has passed all experimental tests. Only the mechanism of electroweak symmetry breaking and of the generation of mass has remained as an unsolved question and is now being addressed with the generalpurpose experiments ATLAS and CMS at the CERN LHC. The discovery of a Standard Model-like scalar Higgs boson of mass of about $125 \mathrm{GeV}$ and first measurements of its decay channels now seem imminent $[1,2]$, which would represent an impressive success of this large technical endeavor. However, the mass of a fundamental scalar particle is affected by large quantum corrections, so that immediately the question of its stabilization with respect to the Planck scale arises.

Weak-scale supersymmetry (SUSY) [3, 4] has long been known to effectively solve not only this "hierarchy problem", but also a considerable number of other problems such as the Grand Unification of gauge symmetries and the question of a viable dark matter candidate. LHC searches for supersymmetric particles have concentrated up to now on the largest, strong production channels, but so far no signs of squarks and gluinos have been detected [5]. Based on LHC searches with about $1 \mathrm{fb}^{-1}$ of data, benchmark points have therefore been defined for future SUSY searches at the LHC [6], and the strong production cross sections and their theoretical uncertainties have been updated to nextto-leading order (NLO) and next-to-leading logarithmic (NLL) accuracy for $p p$ collisions with a center-of-mass energy of $7 \mathrm{TeV}$ [7-12].

In 2012, the LHC center-of-mass energy has increased to $8 \mathrm{TeV}$. In addition, the negative search results for gluino and degenerate squark production and their cascade decays [5] 
imply that the experimental attention now shifts towards third-generation squarks and the weak production channels, in particular the direct production of gauginos. A first investigation of neutralino-chargino pair production and their "golden" decay into three charged leptons has already been published by the ATLAS collaboration with the result that in simplified models such as the constrained Minimal Supersymmetric Standard Model (cMSSM), degenerate lightest chargino $\left(\tilde{\chi}_{1}^{ \pm}\right)$and next-to-lightest neutralino $\left(\tilde{\chi}_{2}^{0}\right)$ masses are excluded up to $300 \mathrm{GeV}$, depending on the mass of the lightest SUSY particle (LSP) assumed to be the lightest neutralino $\tilde{\chi}_{1}^{0}$ [13]. Surprisingly, an independent analysis of ATLAS and CMS measurements of the $W^{+} W^{-}$cross section seems to fit theoretical predictions better when electroweak gauginos of about $100 \mathrm{GeV}$ are included than by the SM cross section alone [14].

The observations above motivate us to update in this paper our precision (NLO+NLL) predictions for direct gaugino pair production. They have been obtained by combining a leading order (LO) calculation [15] and its next-to-leading order corrections [16], which had already been computed independently previously [17], with resummation of the leading and next-to-leading logarithms to all orders in the threshold [16] and small transversemomentum $\left(p_{T}\right)$ [18] regimes or simultaneously in both [19]. A similar program has been carried out for the other weak channel, i.e. the production of sleptons [20-23]. However, their production cross sections are considerably smaller and are not yet accessible at the LHC, so that we leave the corresponding theoretical update for future work. The semiweak associated production of gauginos with squarks [24,25] and gluinos [26-28], which may well become important should the energy available at the LHC prove insufficient for the pair production of the latter, has so far been computed only up to NLO.

In section 2 we present, for a representative selection of benchmark points defined in ref. [6], the total gaugino pair production cross sections and the corresponding scale and parton density function (PDF) uncertainties at the current center-of-mass energy of $8 \mathrm{TeV}$ in tabular form. For comparison and analyses of the data taken previously at $7 \mathrm{TeV}$, we list the corresponding cross sections and uncertainties in appendix A. In addition, an overview of the absolute size of the total cross sections at $8 \mathrm{TeV}$, the impact of the higher-order corrections, and the theoretical uncertainties can be obtained from the figures shown in section 2 illustrating the cross sections as a function of the physical gaugino mass along the model lines attached to the selected benchmark points.

Since the experimental analyses [13] are currently based on LO Monte Carlo simulations of the gaugino signal with parton showers [29, 30], matched [31] to hard tree-level multi-parton corrections [32] and normalized to the NLO total cross section [17], we take the opportunity to analyze in this paper the precision of this procedure. We therefore compare in section 3 invariant-mass and transverse-momentum distributions obtained with parton showers only [30] and matched [31] to one or two additional hard jets [32] to those obtained with threshold [16] and transverse-momentum resummation [18]. Similar comparisons have been presented previously for the hadroproduction of new neutral gauge $\left(Z^{\prime}\right)$ bosons and charged Higgs $\left(H^{ \pm}\right)$bosons in association with top quarks in refs. [33] and $[34,35]$, respectively.

Finally, we summarize our results and present our conclusions in section 4 . 


\section{Total cross sections at $\sqrt{s}=8 \mathrm{TeV}$}

As the ATLAS and CMS experiments are now starting to probe the direct production of gaugino pairs decaying, e.g., into three charged leptons [13], we present in this section an illustrative selection of total gaugino cross sections at the current center-of-mass energy of $\sqrt{s}=8 \mathrm{TeV}$ in order to facilitate precise comparisons with the experimental results. The corresponding cross sections at $\sqrt{s}=7 \mathrm{TeV}$, relevant for data taken in 2010 and 2011, can be found in appendix A. Further results are available from the authors upon request.

\subsection{Benchmark points}

First SUSY search results at the LHC with an integrated luminosity of about $1 \mathrm{fb}^{-1}$ of data excluded six out of the nine SPS benchmark points that had been in use since about a decade $[36,37]$. These cMSSM points therefore had to be replaced by new lines [6] where, motivated by the constraints derived from the measurements of the anomalous magnetic moment of the muon $(g-2)_{\mu}$ and the rare $b \rightarrow s \gamma$ decay, only models with a positive off-diagonal Higgs mixing parameter $\mu>0$ and $\tan \beta=10, A_{0}=0 \mathrm{GeV}$ or $\tan \beta=40$, $A_{0}=-500 \mathrm{GeV}$ were chosen. Here, $\tan \beta$ denotes the ratio of the vacuum expectation values (VEVs) of the neutral components of the two Higgs doublets, and $A_{0}$ is the universal soft trilinear coupling of the Higgs fields to the squarks at the Grand Unification scale. In discussions among the SUSY working groups of the ATLAS and CMS experiments and with the LHC Physics Center at CERN (LPCC) [38], 49 benchmark points lying on these lines were retained for detailed cross section studies [39].

To keep the number of figures manageable, we have selected thirteen out of these 49 points which are included in the two model lines 10.1 and 10.3 with $\tan \beta=10$ and $A_{0}=0 \mathrm{GeV}$ as well as seven additional points located on a line similar to line 40.1 with $\tan \beta=40$ and $A_{0}=-500 \mathrm{GeV}$. Concerning the tables with detailed cross section analyses presented in section 2.2 and the comparison with the Monte Carlo predictions investigated in section 3, we decide to focus in contrast on one specific benchmark scenario for each of the selected lines. The latter are listed in bold face in tables 1, 2 and 3, where we employ the LPCC numbering scheme.

For the first point, with a low value of $\tan \beta=10$ and a vanishing universal trilinear coupling $A_{0}=0 \mathrm{GeV}$, we have chosen an optimistic scenario with low values for the universal scalar mass $m_{0}$ and for the universal gaugino mass $m_{1 / 2}$ at the high scale, the point 1 of the LPCC numbering scheme. These values of $m_{0}$ and $m_{1 / 2}$ lead to modest neutralino and chargino masses at the electroweak scale of about $150-550 \mathrm{GeV}$ (see table 4 in section 2.2). In table 1 we also list, together with the universal scalar and gaugino masses, the physical gluino and average squark masses, which lie in the $1-2 \mathrm{TeV}$ range and are therefore compliant with the current experimental bounds from the ATLAS and CMS experiments. The associated production of the lightest chargino $\tilde{\chi}_{1}^{ \pm}$with the next-to-lightest neutralino $\tilde{\chi}_{2}^{0}$ leads often to the golden trilepton signature. We therefore also present in table 1 the branching ratios of the second lightest neutralino to first and second generation and (s)tau (s)leptons. These quantities indeed allow us to deduce the trilepton production rate from the total cross sections given in the next subsections, since for all the scenarios lying on the 


\begin{tabular}{|c|c||c|c||c|}
\hline Point & $\left(m_{1 / 2}, m_{0}\right)[\mathrm{GeV}]$ & $m_{\tilde{g}}[\mathrm{GeV}]$ & $\left\langle m_{\tilde{q}}\right\rangle[\mathrm{GeV}]$ & $\mathrm{BR}\left(\tilde{\chi}_{2}^{0} \rightarrow \tilde{\ell} \ell / \tilde{\tau} \tau\right)[\%]$ \\
\hline $\mathbf{1}$ & $\mathbf{4 0 0 , \mathbf { 1 0 0 } )}$ & $\mathbf{9 3 5}$ & $\mathbf{8 4 0}$ & $\mathbf{1 6} / \mathbf{2 2}$ \\
2 & $(450,112.5)$ & 1040 & 940 & $20 / 20$ \\
3 & $(500,125)$ & 1145 & 1030 & $24 / 19$ \\
4 & $(550,137.5)$ & 1255 & 1125 & $26 / 18$ \\
5 & $(600,150)$ & 1355 & 1220 & $28 / 18$ \\
6 & $(650,162.5)$ & 1460 & 1310 & $28 / 17$ \\
7 & $(700,175)$ & 1565 & 1405 & $29 / 17$ \\
\hline
\end{tabular}

Table 1. Selection of benchmark points on the cMSSM model line 10.1 of ref. [6] with $\tan \beta=10$, $A_{0}=0 \mathrm{GeV}$, and $m_{0}=0.25 \times m_{1 / 2}$. The points are spaced in steps of $\Delta m_{1 / 2}=50 \mathrm{GeV}$, and the gluino and average squark masses are rounded to $5 \mathrm{GeV}$ accuracy. We also present the branching ratios of the next-to-lightest neutralino into $\ell=e, \mu$ and $\tau$ (s)leptons.

\begin{tabular}{|c||c||c|c||c|}
\hline Point & $\left(m_{1 / 2}, m_{0}\right)[\mathrm{GeV}]$ & $m_{\tilde{g}}[\mathrm{GeV}]$ & $\left\langle m_{\tilde{q}}\right\rangle[\mathrm{GeV}]$ & $\mathrm{BR}\left(\tilde{\chi}_{2}^{0} \rightarrow \tilde{\chi}_{1}^{0} h\right)[\%]$ \\
\hline 15 & $(375,250)$ & 885 & 825 & 89 \\
16 & $(450,300)$ & 1050 & 975 & 92 \\
17 & $(525,350)$ & 1210 & 1125 & 92 \\
$\mathbf{1 8}$ & $(\mathbf{6 0 0}, \mathbf{4 0 0})$ & $\mathbf{1 3 7 0}$ & $\mathbf{1 2 7 5}$ & $\mathbf{9 2}$ \\
19 & $(675,450)$ & 1525 & 1420 & 92 \\
20 & $(750,500)$ & 1680 & 1565 & 92 \\
\hline
\end{tabular}

Table 2. Selection of benchmark points on the cMSSM model line 10.3 of ref. [6] with $\tan \beta=10$, $A_{0}=0 \mathrm{GeV}$, and $m_{1 / 2}=1.5 \times m_{0}$. The points are spaced in steps of $\Delta m_{0}=50 \mathrm{GeV}$, and the gluino and average squark masses are rounded to $5 \mathrm{GeV}$ accuracy. We also present the branching ratio of the dominant decay mode of the next-to-lightest neutralino into a Higgs boson.

line 10.1 the lightest chargino $\tilde{\chi}_{1}^{ \pm}$and the sleptons always decay (in a first approximation) to a single observable lepton and missing transverse energy.

Our second benchmark scenario consists in the point 18 lying on the model line 10.3. As for the point 1 , the value of the ratio of the Higgs VEVs has been set to $\tan \beta=10$ and the universal trilinear scalar coupling is vanishing at high energies. In contrast to the first scenario, we rather adopt here higher values for the universal scalar and gaugino masses $m_{0}$ and $m_{1 / 2}$, which yields slightly heavier neutralino and chargino states with masses of about 250-770 GeV (see table 5 in section 2.2). As for the first scenario, this combination of values for $m_{0}$ and $m_{1 / 2}$ shifts the masses of the squarks and gluino to the 1-2 $\mathrm{TeV}$ range as shown in table 2 , rendering the scenarios of the line 10.3 not (yet) excluded by LHC data. As shown in the last column of table 2, the next-to-lightest neutralino decays most of the time to a Higgs boson, since as an almost pure light wino it can not decay into the heavier superpartners of the left-handed leptons and quarks. As another consequence of the heavy squark masses, $t$ - and $u$-channel squark exchanges leading to the production of neutralino and gaugino pairs are suppressed by heavy propagators. One however expects reasonable production rates for final states containing one or two neutralinos $\tilde{\chi}_{2}^{0}$ via dominant $s$-channel weak boson exchanges and reduced destructive interferences with the $t$ - and $u$-channel diagrams 


\begin{tabular}{|c||c||c|c||c|}
\hline Point & $\left(m_{1 / 2}, m_{0}\right)[\mathrm{GeV}]$ & $m_{\tilde{g}}[\mathrm{GeV}]$ & $\left\langle m_{\tilde{q}}\right\rangle / m_{\tilde{t}_{1}} / m_{\tilde{b}_{1}}[\mathrm{GeV}]$ & $\operatorname{BR}\left(\tilde{\chi}_{2}^{0} \rightarrow \tilde{\tau}_{1} \tau / h \tilde{\chi}_{1}^{0}\right)[\%]$ \\
\hline 27 & $(400,300)$ & 940 & $890 / 615 / 745$ & $98 / 2$ \\
28 & $(450,325)$ & 1050 & $985 / 700 / 835$ & $97 / 3$ \\
29 & $(500,350)$ & 1155 & $1185 / 780 / 925$ & $96 / 4$ \\
30 & $(550,375)$ & 1260 & $1180 / 860 / 1010$ & $95 / 4$ \\
$\mathbf{3 1}$ & $(\mathbf{6 0 0 , 4 0 0 )}$ & $\mathbf{1 3 6 5}$ & $\mathbf{1 2 7 5} / \mathbf{9 4 0} / \mathbf{1 1 0 0}$ & $\mathbf{9 5 / 5}$ \\
32 & $(650,425)$ & 1470 & $1370 / 1020 / 1185$ & $94 / 5$ \\
33 & $(700,450)$ & 1575 & $1465 / 1095 / 1275$ & $94 / 6$ \\
\hline
\end{tabular}

Table 3. Selection of benchmark points on a cMSSM model line similar to line 40.1 of ref. [6] with $\tan \beta=40, A_{0}=-500 \mathrm{GeV}$, and $m_{0}=0.5 \times m_{1 / 2}+100 \mathrm{GeV}$. The points are spaced in steps of $\Delta m_{1 / 2}=50 \mathrm{GeV}$. The mass of the gluino, the average mass of the heavier squarks, and the masses of the (considerably lighter) top and bottom squarks are rounded to $5 \mathrm{GeV}$ accuracy. We also present the branching ratios for the decays of the next-to-lightest neutralino into $\tau$ (s)leptons and Higgs bosons.

(in contrast to a light squark scenario). Since the lightest chargino decays with a $98 \%$ branching ratio to a $W$-boson, one can deduce the production rate of golden signatures from the values of the $\tilde{\chi}_{2}^{0} \tilde{\chi}_{1}^{ \pm}$total cross section presented in section 2.2 and section 2.3 after accounting for the branching ratio of a Higgs boson decaying to a pair of tau leptons and the rate of leptonic $W$-boson decays.

Our third selected scenario lies on a model line similar to the line 40.1 of ref. [6] with a large value of $\tan \beta=40$ and a large negative value of the universal scalar trilinear coupling $A_{0}=-500 \mathrm{GeV}$. We retain the high values of the universal scalar and gaugino masses that have driven the choice of our second scenario and therefore adopt the point 31 of the LPCC numbering scheme as our third benchmark point. Comparing with the point 18 , the neutralino and chargino masses are not drastically affected by the different choices of $\tan \beta$ and $A_{0}$ and hence lie in the $250-825 \mathrm{GeV}$ range (see table 6 in section 2.2). Similarly, the gluino and first and second generation squark masses are not drastically affected either as presented in table 3 . In contrast, the large values of $\tan \beta$ and $A_{0}$ induce an important mixing among the third-generation squark interaction eigenstates (and to a smaller extent among the left- and right-handed stau states). As a consequence, the masses of the lightest stop and sbottom are much lower than the average squark mass as shown in table 3, and these states are almost maximal admixtures of the left-handed and righthanded third generation squark eigenstates. We also present the two main decay modes of the second neutralino, decaying mainly to an associated pair of stau (always decaying to a tau and a lightest neutralino) and tau lepton but also, at a much smaller rate, to a Higgs boson and missing energy carried by the lightest neutralino. As for the two other selected scenarios, the cross section corresponding to the trilepton mode can be deduced from the values of the total cross sections given in section 2.2 and section 2.3 and from the branching ratios of the lightest Higgs boson to taus as well as the one associated to tau leptonic decays. 
We emphasize that while the increasing experimental limits on the squark and gluino masses might exclude the corresponding cMSSM scenarios, the (lower) gaugino masses may still remain allowed and their total cross sections therefore approximately valid in the context of more general SUSY-breaking scenarios.

\subsection{Total cross sections for the benchmark points 1, 18, and 31}

The production of pairs of charginos and neutralinos has been initially studied at leading order of perturbative QCD in the early 1980s [40, 41], while more recently polarization [15, 42] and flavor-violating [43-45] effects have been included and investigated. Next-to-leading order corrections have been supplemented to the leading order approximation $[16,17]$ and have been found to be important, in particular due to the presence of large logarithmic contributions arising from soft and collinear parton emission by the initial state particles. Since they spoil the convergence of the perturbative series, these logarithms have to be resummed to all orders in the strong coupling constant to allow for reliable theoretical predictions in the entire phase space, including the regions related to soft and collinear QCD radiation. Transverse-momentum and threshold resummation have then been achieved at the next-to-leading logarithmic accuracy independently [16, 18] and simultaneously [19].

In this section, we present in tables 4,5 , and 6 total production cross sections for various combinations of neutralino and chargino pairs in the context of the benchmark points 1, 18 and 31 introduced in section 2.1 at the leading and next-to-leading order of perturbative QCD as well as after matching the NLO results to threshold resummation. The masses of the produced gauginos are also given as references, while the values of the squark masses entering the partonic cross section can be found in tables 1, 2 and 3 .

The LO results, given in the fourth column of the tables, are computed as in ref. [15] after convolving (unpolarized) partonic cross sections with the LO set of the MSTW 2008 parton densities [46], accounting for five light flavors of massless quarks and as agreed among the SUSY working groups of ATLAS, CMS and the LPCC. We use a top quark mass of $173.1 \mathrm{GeV}$ [47] and the values of $m_{Z}=91.1876 \mathrm{GeV}$ and $m_{W}=80.403 \mathrm{GeV}$ [48] for the masses of the electroweak gauge bosons, and we consider the CKM matrix as the identity matrix in flavor space. The supersymmetric spectra have been generated with the SuSpect 2.41 program [49], allowing to obtain low-energy supersymmetric masses and parameters from universal parameters defined at the Grand Unification scale and evolved down through renormalization group running at the two-loop level. The central values of the cross sections in the tables have been computed after fixing the factorization scale $\mu_{F}$ to the average final state particle mass, while the uncertainties are estimated after multiplying the central value of the factorization scale by a factor lying in the 0.5-2 range.

For our NLO predictions, we follow the detailed computations preformed in our previous work [16], where both the QCD and the SUSY-QCD contributions are included in the virtual pieces of the cross section, and give our results in the fifth column of the tables. We emphasize that we also consider, as in our earlier calculations, internal squark mixing, i.e., the squarks are kept non-degenerate in the loops. The partonic cross sections are convolved this time with the MSTW 2008 NLO parton density sets [46], and the central values of 


\begin{tabular}{|c|c|c|c|c|c|}
\hline Process & $m_{1}[\mathrm{GeV}]$ & $m_{2}[\mathrm{GeV}]$ & LO [fb] & NLO [fb] & $\mathrm{NLO}+\mathrm{NLL}[\mathrm{fb}]$ \\
\hline$p p \rightarrow \chi_{1}^{0} \chi_{1}^{0}$ & 161.7 & 161.7 & $0.81_{-5.3 \%}^{+5.8 \%}$ & $\begin{array}{l}1.06_{-3.0 \%-2.0 \%}^{+3.5 \%+2.8 \%} \\
\end{array}$ & $\begin{array}{l}1.03_{-0.6 \%-2.0 \%}^{+0.5 \%+2.9 \%} \\
\end{array}$ \\
\hline$p p \rightarrow \chi_{1}^{0} \chi_{1}^{-}$ & 161.7 & 303.5 & $0.16_{-5.5 \%}^{+6.0 \%}$ & $0.20_{-2.4 \%-2.4 \%}^{+2.5 \%+2.9 \%}$ & $0.20_{-0.3 \%-2.5 \%}^{+0.0 \%+2.9 \%}$ \\
\hline$p p \rightarrow \chi_{2}^{0} \chi_{2}^{0}$ & 303.8 & 303.8 & $0.85_{-7.9 \%}^{+9.2 \%}$ & $1.07_{-3}^{+3}$ & $1.05_{-0.4 \%}^{+0.0 \%}$ \\
\hline$p p \rightarrow \chi_{2}^{0} \chi_{3}^{0}$ & 303.8 & 526.5 & $0.21_{-8.1}^{+9.4}$ & $0.25_{-2.9 \%}^{+2.6 \%}$ & $0.25_{-0.5 \%-2.3 \%}^{+0.1 \%+3.2 \%}$ \\
\hline$p p \rightarrow \chi_{2}^{0} \chi_{1}^{-}$ & 303.8 & 303.5 & $14.46_{-6.1 \%}^{+6.7 \%}$ & $17.25_{-1.7 \%-2.6 \%}^{+1.6 \%+3.0 \%}$ & $17.05_{-0.7 \%-2.6 \%}^{+0.2 \%+3.1 \%}$ \\
\hline$p p \rightarrow \chi_{3}^{0} \chi_{4}^{0}$ & 526.5 & 542.4 & $0.83_{-9.3 \%}^{+11.0 \%}$ & $0.97_{-3 .}^{+2 .}$ & $0.96_{-0.9 \%}^{+0.4 \%}$ \\
\hline$p p \rightarrow \chi_{3}^{0} \chi_{1}^{-}$ & 526.5 & 303.5 & $0.12_{-8.1 \%}^{+9.4 \%}$ & $0.15_{-2.9 \%}^{+2.6 \%}$ & $0.15_{-0.6 \%-3}^{+0.1 \%+3}$ \\
\hline$p p \rightarrow \chi_{3}^{0} \chi_{2}^{-}$ & 526.5 & 542.2 & $0.42_{-9.5 \%}^{+11.2 \%}$ & $0.50_{-3.3 \%}^{+2.8 \%}$ & $0.49_{-0.9 \%}^{+0.4 \%}$ \\
\hline$p p \rightarrow \chi_{4}^{0} \chi_{2}^{-}$ & 542.4 & 542.2 & $0.39_{-9.6 \%}^{+11.3 \%}$ & $0.47_{-3.2 \%}^{+2.7 \%}$ & $0.46_{-1.1 \%}^{+0.5 \%+}$ \\
\hline$p p \rightarrow \chi_{1}^{+} \chi_{1}^{0}$ & 303.5 & 161.7 & $0.38_{-5.4 \%}^{+6.0 \%}$ & $0.46_{-2.4 \%-2}^{+2.5 \%+}$ & $0.46_{-0.5 \%}^{+0.2 \%+}$ \\
\hline$p p \rightarrow \chi_{1}^{+} \chi_{2}^{0}$ & 303.5 & 303.8 & $35.16_{-5.8 \%}^{+6.3 \%}$ & $40.90_{-1.7 \%-2.2 \%}^{+1.6 \%+2.9 \%}$ & $40.51_{-0.3 \%-2.2 \%}^{+0.0 \%+2.9 \%}$ \\
\hline$p p \rightarrow \chi_{1}^{+} \chi_{3}^{0}$ & 303.5 & 526.5 & $0.34_{-7.9 \%}^{+9.2 \%}$ & $0.40_{-2.9 \%-2}^{+2.6 \%+3}$ & $0.40_{-0.3 \%-2.5 \%}^{+0.0 \%+3.6 \%}$ \\
\hline$p p \rightarrow \chi_{1}^{+} \chi_{1}^{-}$ & 303.5 & 303.5 & $25.64_{-5.9 \%}^{+6.6 \%}$ & $30.37_{-1.9 \%-2.0 \%}^{+1.7 \%+2.7 \%}$ & $30.04_{-0.5 \%-2.1 \%}^{+0.0 \%+2.7 \%}$ \\
\hline$p p \rightarrow \chi_{2}^{+} \chi_{3}^{0}$ & 542.2 & 526.5 & $1.27_{-9.4 \%}^{+11.1 \%}$ & $1.46_{-3.3 \%-2.7 \%}^{+2.9 \%+4.4 \%}$ & $1.45_{-0.7 \%-2.9 \%}^{+0.3 \%+4.3 \%}$ \\
\hline$p p \rightarrow \chi_{2}^{+} \chi_{4}^{0}$ & 542.2 & 542.4 & $1.21_{-9.5 \%}^{+11.2 \%}$ & $1.37_{-3.2 \%-2.8 \%}^{+2.7 \%}+4.4 \%$ & $1.36_{-0.8 \%-2.6 \%}^{+0.4 \%+4.6 \%}$ \\
\hline$p p \rightarrow \chi_{2}^{+} \chi_{2}^{-}$ & 542.2 & 542.2 & $0.86_{-9.3 \%}^{+10.9 \%}$ & $\begin{array}{l}1.00_{-3.1 \%-2.4 \%}^{+2.6 \%}+4.0 \% \\
\end{array}$ & $\begin{array}{l}0.99_{-0.9 \%-2.4 \%}^{+0.4 \%+4.1 \%} \\
\end{array}$ \\
\hline
\end{tabular}

Table 4. Total cross sections related to the production of various gaugino pairs of masses $m_{1}$ and $m_{2}$, presented together with the associated scale and PDF uncertainties for the LHC running at a center-of-mass energy of $\sqrt{s}=8 \mathrm{TeV}$ in the context of the benchmark point 1 of the LPCC numbering scheme. The cross sections are given at the leading order and next-to-leading order of perturbative QCD and matched to threshold resummation. The PDF uncertainties are not shown for the LO results. Any cross section smaller than $0.1 \mathrm{fb}$ is omitted.

\begin{tabular}{|c|c|c|c|c|c|}
\hline Proce & $m_{1}[\mathrm{GeV}]$ & $m_{2}[\mathrm{GeV}]$ & LO [fb] & $\mathrm{NLO}[\mathrm{fb}]$ & $\mathrm{NLO}+\mathrm{NLL}[\mathrm{fb}]$ \\
\hline$p p \rightarrow \chi_{1}^{0} \chi_{1}^{0}$ & 249.6 & 249.6 & $0.13_{-7.5 \%}^{+8.6 \%}$ & $0.16_{-3.4 \%}^{+3.5 \%+}$ & $0.16_{-0.3 \%-2.4 \%}^{+0.2 \%+3.5 \%}$ \\
\hline$p p \rightarrow \chi_{2}^{0} \chi_{1}^{-}$ & 471.9 & 471.8 & $1.63_{-\varepsilon}^{+1}$ & $1.88_{-2}^{+1}$ & $1.86_{-1}^{+0}$ \\
\hline$p p \rightarrow \chi_{1}^{+} \chi_{2}^{0}$ & 471.8 & 471.9 & $4.73_{-8}^{+\mathrm{S}}$ & $5.28_{-2}^{+1}$ & $5.22_{-0.6 \%}^{+0.3 \%}$ \\
\hline$p p \rightarrow \chi_{1}^{+} \chi_{1}^{-}$ & 471.8 & 471.8 & $3.13_{-8.4 \%}^{+9.8 \%}$ & $3.57_{-2}^{+1}$ & $3.52_{-0.7 \%-2.3 \%}^{+0.4 \%+3.7 \%}$ \\
\hline$p p \rightarrow \chi_{2}^{+} \chi_{3}^{0}$ & 766.3 & 754.0 & $0.16_{-11.6 \%}^{+14.2 \%}$ & $0.17_{-4}^{+3}$ & $0.17_{-1.8 \%-3.8 \%}^{+1.0 \%+6.1 \% \%}$ \\
\hline$p p \rightarrow \chi_{2}^{+} \chi_{4}^{0}$ & 766.3 & 766.6 & $0.15_{-11.7 \%}^{+14.3 \%}$ & $0.16_{-4.2}^{+3.4}$ & $0.16_{-1.8 \%-4.0 \%}^{+1.1 \%+6.1 \%}$ \\
\hline$p p \rightarrow \chi_{2}^{+} \chi_{2}^{-}$ & 766.3 & 766.3 & $0.11_{-11.2 \%}^{+13.6 \%}$ & $0.12_{-3.9 \%-3.5 \%}^{+3.1 \%+6.0 \%}$ & $0.12_{-1.8 \%-3.6 \%}^{+1.0 \%+6.0 \%}$ \\
\hline
\end{tabular}

Table 5. Same as table 4 for the benchmark point 18 of the LPCC numbering scheme.

the total cross sections are given in the tables together with the theoretical uncertainties related to scale variation and the choice of the PDF set. Scale uncertainties are derived following the standard approach of simultaneously varying the factorization scale $\mu_{F}$ and renormalization scale $\mu_{R}$ by multiplying and dividing the average final-state particle mass by a factor of two. The PDF errors are obtained by evaluating the envelope of the cross section when employing the $68 \%$ confidence level range of the MSTW 2008 parton densi- 


\begin{tabular}{|c||l|l||l|l|l|}
\hline Process & $m_{1}[\mathrm{GeV}]$ & $m_{2}[\mathrm{GeV}]$ & LO [fb] & NLO [fb] & NLO+NLL [fb] \\
\hline$p p \rightarrow \chi_{1}^{0} \chi_{1}^{0}$ & 251.7 & 251.7 & $0.12_{-7.5 \%}^{+8.6 \%}$ & $0.16_{-3.4 \%-2.3 \%}^{+3.5 \%}$ & $0.15_{-0.3 \%}^{+0.2 \%+3.4 \%}$ \\
$p p \rightarrow \chi_{2}^{0} \chi_{1}^{-}$ & 478.5 & 478.5 & $1.50_{-8.1 \%}^{+10.6 \%}$ & $1.73_{-2.8 \%}^{+1.8 \%+4.2 \%}$ & $1.71_{-1.6 \%}^{+0.6 \%+4.1 \%}$ \\
$p p \rightarrow \chi_{1}^{+} \chi_{2}^{0}$ & 478.5 & 478.5 & $4.37_{-8.5 \%}^{+9.9 \%}$ & $4.86_{-2.8 \%}^{+1.8 \%}+3.9 \%$ & $4.81_{-0.6 \%}^{+0.3 \%+4.2 \%}$ \\
$p p \rightarrow \chi_{1}^{+} \chi_{1}^{-}$ & 478.5 & 478.5 & $2.89_{-8.5 \%}^{+9.9 \%}$ & $3.28_{-2.5 \%}^{+1.9 \%+3.5 \%}$ & $3.24_{-0.5 \%}^{+0.5 \%+3 \%} \%$ \\
\hline
\end{tabular}

Table 6. Same as table 4 for the benchmark point 31 of the LPCC numbering scheme.

ties. The (asymmetric) errors $\Delta \sigma_{\text {up }}$ and $\Delta \sigma_{\text {down }}$ are computed as defined by the MSTW collaboration, i.e., according to

$$
\begin{aligned}
\left(\Delta \sigma_{\mathrm{up}}\right)^{2} & =\sum_{k=1}^{n}\left\{\max \left[\sigma_{k}^{+}-\sigma_{0}, \quad \sigma_{k}^{-}-\sigma_{0}, \quad 0\right]\right\}^{2}, \\
\left(\Delta \sigma_{\text {down }}\right)^{2} & =\sum_{k=1}^{n}\left\{\max \left[\sigma_{0}-\sigma_{k}^{+}, \quad \sigma_{0}-\sigma_{k}^{-}, \quad 0\right]\right\}^{2},
\end{aligned}
$$

where $\sigma_{0}$ consists in the value of the cross section computed when using the central set of parton densities, while $\sigma_{k}^{+}$and $\sigma_{k}^{-}$are those obtained from $\pm 1 \sigma$ variations along the $k^{\text {th }}$ eigenvector of the covariance matrix associated to the PDF fit.

In the last column of the tables, we present the total cross section after having matched the next-to-leading order results with threshold resummation at the next-to-leading logarithmic accuracy. After performing a Mellin transformation, the cross section can be written in the Mellin $N$-space as a simple product between the parton densities $f_{a / p}$ and $f_{b / p}$ and the partonic cross section $\sigma_{a b}$,

$$
M^{2} \frac{\mathrm{d} \sigma}{\mathrm{d} M^{2}}(N-1)=\sum_{a b} f_{a / p}\left(N, \mu^{2}\right) f_{b / p}\left(N, \mu^{2}\right) \sigma_{a b}\left(N, M^{2}, \mu^{2}\right),
$$

where we have set $\mu_{F}=\mu_{R}=\mu$ for brevity and where $M$ denotes the invariant mass of the produced gaugino pair. Within the threshold resummation formalism, the partonic cross section can be refactorized into a closed exponential form as [50]

$$
\sigma_{a b}\left(N, M^{2}, \mu^{2}\right)=\mathcal{H}_{a b}\left(M^{2}, \mu^{2}\right) \exp \left[\mathcal{G}_{a b}\left(N, M^{2}, \mu^{2}\right)\right]+\mathcal{O}\left(\frac{1}{N}\right)
$$

The function $\mathcal{H}$ can be perturbatively computed, describes the hard part of the scattering process, and is independent of the Mellin variable $N$. The function $\mathcal{G}$ collects soft and collinear parton emission and absorbs the large logarithmic contributions arising at fixed order. We then further improve the resummation formula of eq. (2.3) above by including in the $\mathcal{H}$-function the dominant $1 / N$-terms stemming from universal collinear radiation of the initial state partons [51-54]. The exact expressions of the coefficients of the expansion of the two functions $\mathcal{H}$ and $\mathcal{G}$ at the next-to-leading logarithmic accuracy can be found in ref. [16].

The resummed predictions are known to be valid near production threshold, where the logarithmic terms of the cross section dominate, in contrast to the fixed order computations, which are assumed to be valid far from this threshold. Therefore, in order to 
get reliable predictions in all kinematic regions, both results are consistently matched by summing the resummed $\sigma_{a b}^{\text {(res.) }}$ and the fixed order $\sigma_{a b}^{\text {(f.o.) }}$ cross sections and subtracting their overlap $\sigma_{a b}^{\text {(exp.) }}$,

$$
\sigma_{a b}=\sigma_{a b}^{(\text {res. })}+\sigma_{a b}^{(\text {f.o. })}-\sigma_{a b}^{(\text {exp. })} .
$$

We again refer to ref. [16] for the exact expression of $\sigma_{a b}^{\text {(exp.) }}$ which is, for a NLO+NLL matching, the $\mathcal{O}\left(\alpha_{s}\right)$ series expansion of the resummed cross section of eq. (2.3). Since the two quantities $\sigma_{a b}^{\text {(res.) }}$ and $\sigma_{a b}^{\text {(exp.) }}$ are computed in Mellin space, an inverse transform must be performed in order to obtain the total cross section in terms of physical quantities

$$
\begin{aligned}
M^{2} \frac{\mathrm{d} \sigma}{\mathrm{d} M^{2}}(\tau)= & \frac{1}{2 \pi i} \sum_{a b} \int_{\mathcal{C}_{N}} \mathrm{~d} N \tau^{-N} f_{a / p}\left(N+1, \mu^{2}\right) f_{b / p}\left(N+1, \mu^{2}\right) \\
& \times\left[\sigma_{a b}^{(\text {res. })}\left(N+1, M^{2}, \mu^{2}\right)-\sigma_{a b}^{(\text {(exp. })}\left(N+1, M^{2}, \mu^{2}\right)\right],
\end{aligned}
$$

where $\tau=M^{2} / s$. To avoid singularities in the integrand related to the Landau pole of the strong coupling constant and to the Mellin moments of the parton densities at small momentum fraction, we choose an integration contour $\mathcal{C}_{N}$ according to the principal value procedure [55] and the minimal prescription [56].

The resummed results are given in tables 4, 5 and 6 together with the uncertainties derived from scale variations and deviations from the central fit of the MSTW 2008 parton densities. The latter are computed as for the pure NLO results and described above.

One observes, by investigating the numerical results, an improvement of the stability of the perturbative series, as the scale dependence of the total cross section is tamed by the resummation of the soft and collinear radiation contributions. At LO, the factorization scale $\mu_{F}$ appearing within the evolution of the parton densities already induces large logarithmic terms yielding an uncertainty of about $10 \%$ on the cross section. This effect is then attenuated at NLO, but one gets an additional explicit dependence in the renormalization scale $\mu_{R}$ through the strong coupling constant and the loop contributions. After matching the NLO results with threshold resummation at NLL, the scale variations are eventually considerably reduced thanks to the inclusion of dominant higher order contributions within the Sudakov form factor $\mathcal{G}$. In contrast, the uncertainties related to the choice of the employed parton densities coincide when comparing the NLO and the NLO+NLL results, which is not surprising since the same PDF sets enter both computations.

\subsection{Total cross sections for model lines 10.1, 10.3, and 40.1}

In order to give a more complete overview of the absolute size of the total gaugino cross sections, we present in this section the total cross sections for the golden trilepton channel, i.e. the production of a $\tilde{\chi}_{2}^{0} \tilde{\chi}_{1}^{+}$pair, as a function of their almost equal mass $m_{\tilde{\chi}}$ at the LHC with a center-of-mass energy of $8 \mathrm{TeV}$

The results for model line 10.1 are shown in figure 1 at LO (dotted), NLO (dashed), and NLL+NLO (full). As the soft SUSY-breaking mass $m_{1 / 2}$ increases from 400 to $700 \mathrm{GeV}$, the gaugino masses rise from 300 to $550 \mathrm{GeV}$. Consequently, the total cross section falls by over an order of magnitude from about $40 \mathrm{fb}$ to about $2 \mathrm{fb}$. The impact of the NLO 


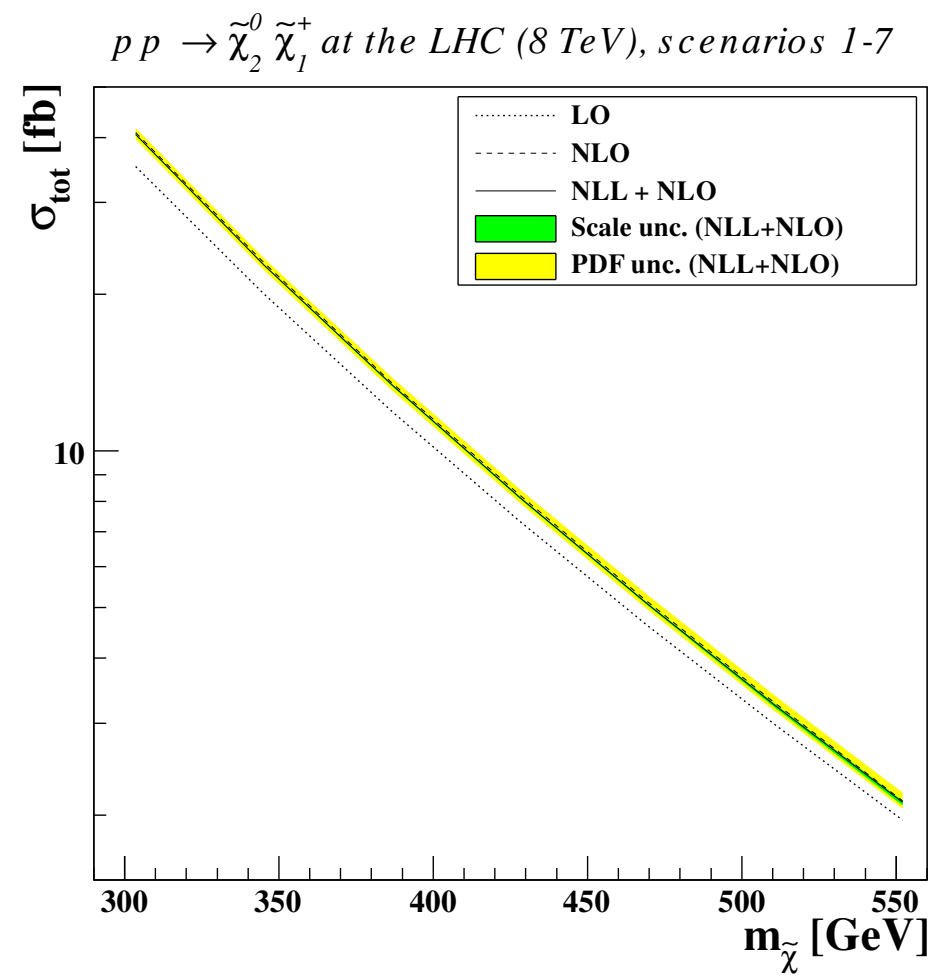

Figure 1. The total cross section at LO (dotted), NLO (dashed), and NLO+NLL (full) with its scale (green) and PDF (yellow) uncertainty for the production of a $\chi_{2}^{0} \chi_{1}^{+}$pair as a function of their almost degenerate mass $m_{\tilde{\chi}}$ at the LHC with $\sqrt{s}=8 \mathrm{TeV}$ and benchmark points 1-7.

corrections to the LO prediction is clearly visible, and the cross section is then stabilized at NLL+NLO, where it remains almost unchanged with respect to the NLO result, but is considerably less scale-dependent. The overall theoretical uncertainty is thus dominated by the PDF uncertainty, estimated as described in the previous section. Similar results are obtained for model lines 10.3 and 40.1 (not shown), as they have similar physical gaugino mass ranges.

\section{Comparisons of invariant-mass and transverse-momentum distribu- tions}

Typical final-state signatures associated to the production of the electroweak superpartners of the Standard Model gauge and Higgs bosons contain in general abundant initial state QCD radiation. The latter has important effects on the shapes of the kinematical distributions, in particular due to the large logarithmic contributions arising in the phase space regions where these additional partons are neither widely separated nor hard. In this case, reliable theoretical predictions require a consistent reorganization of the logarithmic terms so that they are effectively resummed to all orders in the strong coupling constant and embedded in the so-called Sudakov form factor. In the resummation computations presented in section 2, this factor has been derived at the next-to-leading logarithmic accu- 
racy from the knowledge of the perturbative expansion of the hard-scattering function at the next-to-leading order. This leads to an accurate description of the QCD environment in phase space regions where radiation is soft and/or collinear. In order to obtain a good description over the whole kinematical range, including also the hard radiation regime, the resummed results have to be eventually matched to a fixed order calculation describing precisely the hard emission.

In experimental analyses or phenomenological investigations relying on Monte Carlo simulations, the production of additional jets is traditionally simulated using parton showering programs, such as HeRwig $[29,57]$ or Pythia [30, 58], which describe QCD emissions as successive branchings of a mother parton into two daughter partons. These tools are based on Markov chain techniques built upon the Sudakov form factor dictating the probability laws for a specific parton to radiate or not. In contrast to the resummation techniques adopted for the predictions shown in section 2, where the Sudakov form factor is computed at the next-to-leading logarithmic accuracy, the parton showering tools use only a leading-logarithmic precision, sometimes including partial (but not complete) next-to-leading logarithmic contributions. The reason is that only such approximations allow for a description in terms of a Monte Carlo algorithm. Moreover, for supersymmetric processes the hard scattering can only be evaluated at tree level, since no SUSY process has so far been implemented into NLO Monte Carlo tools such as MC@NLO [59, 60] or Powheg [61]. This description is formally only correct in the soft and collinear radiation regions of the phase space, and it fails when considering the production of hard and widely separated additional partons due to missing subleading terms. In this case, matrix elements describing the same final state together with an additional parton are required.

The matrix-element approach is indeed accurate for describing hard and widely separated emissions, but is known to break down in the soft and collinear radiation limits. Contrary, parton showering gives a proper description in the soft and collinear kinematical regions, but underestimates hard emissions. Therefore, several matching algorithms, such as the Catani-Krauss-Kuhn-Webber (CKKW) scheme based on event reweighting [62, 63] or the Mangano (MLM) scheme based on event rejection [64], have been developed to combine the two methods in a consistent way. Hence this avoids the possible double counting arising from radiations which can be taken into account both at the level of the matrix element and at the level of the Sudakov form factor. These different procedures have been extensively applied, compared and confronted to the data in the context of various Standard Model processes [65-68] and also used for recent Beyond the Standard Model explorations [69-71].

In this section, we present the first comparison between resummation (matched to fixed order), next-to-leading order and parton showering (matched to matrix elements) predictions in the context of chargino and neutralino production. We focus on the distributions of the transverse momentum $\left(p_{T}\right)$ and invariant mass $(M)$ of the superparticle pairs produced at the LHC collider, running at a center-of-mass energy of $8 \mathrm{TeV}$. Concerning the most precise predictions, we employ the transverse-momentum and threshold resummation formalisms. Threshold resummation has been briefly described in section 2.2 and more details can be found in ref. [16]. As for the threshold case (see eq. (2.2)), resummation in 
the transverse-momentum regime is also performed in Mellin space. The partonic cross is computed in the impact parameter $b$-space

$$
\sigma_{a b}\left(p_{T}^{2}, M^{2}, \mu^{2}\right)=\frac{M^{2}}{s} \int_{0}^{\infty} \mathrm{d} b \frac{b}{2} J_{0}\left(b p_{T}\right) \sigma_{a b}\left(b, N, M^{2}, s, \mu^{2}\right),
$$

where the function $J_{0}$ is the $0^{\text {th }}$-order Bessel function, so that the integrand can be refactorized under a closed exponential form, as in eq. (2.3) for threshold resummation,

$$
\sigma_{a b}\left(b, N, M^{2}, \mu^{2}\right)=\mathcal{H}_{a b}\left(N, M^{2}, \mu^{2}\right) \exp \left[\mathcal{G}_{a b}\left(b, N, M^{2}, \mu^{2}\right)\right]+\mathcal{O}\left(\frac{1}{N}\right) .
$$

As usual, the Sudakov form factor $\mathcal{G}$ contains the soft and collinear radiation contributions and $\mathcal{H}$ is the hard function, independent of the impact parameter $b$. More details and the exact expressions of these functions, evaluated at the next-to-leading logarithmic accuracy, can be found in ref. [18]. Matching to the fixed order computations and the inverse Mellin transform to get back to the physical space are eventually performed, as sketched in eqs. (2.4) and (2.5).

Concerning the merging of the parton showering with the hard-scattering matrix elements, we follow the $k_{T}$-MLM matching scheme [69] as implemented in the MADGRAPHMadEvent event generator version 5 [32], interfaced to Pythia 6 [30]. The UFO [72] model files required for MADGRAPH are generated starting from the MSSM implementation in the FEYNRULES package [73-75] after loading the supersymmetric spectrum associated to the benchmark points 1, 18 and 31 described in section 2 and obtained as in section 2.2 with the SuSpect 2.41 program [49]. We generate three distinct parton-level event samples for final states containing, in addition to the pair of supersymmetric particles, zero, one and two extra partons, respectively. We subsequently merge them after parton-showering, employing the $k_{T}$-MLM matching scheme. The (parton-level) jets are generated with a minimum jet measure $k_{T}$ of $50 \mathrm{GeV}$ between two final state partons $i$ and $j$, where $k_{T}$ is defined by

$$
k_{T}^{2}=\min \left(p_{T i}^{2}, p_{T j}^{2}\right) R_{i j} .
$$

In the equation above, the quantities $p_{T i}$ and $p_{T j}$ are the transverse momenta of the two partons under consideration and $R_{i j}$ denotes their angular distance in the $(\eta, \phi)$ plane. In the case where one of the two partons is an initial state parton, we define the jet measure as the transverse momentum of the final state parton

$$
k_{T}=p_{T i}
$$

and require its value to be larger than $20 \mathrm{GeV}$, which ensures better QCD factorization properties with respect to initial-state collinear singularities [76]. The events are then passed to PYTHIA for showering, and jets are reconstructed using FASTJET [77] in the $k_{T}$-jet algorithm with a cut-off scale $Q^{\text {match }}$ set to $70 \mathrm{GeV}$. The jets are said to be matched to one of the original partons if the $k_{T}$-measure between the jet and the parton is smaller than $Q^{\text {match }}$. The events are selected only if each jet is matched to one parton, with the exception of the two-jet sample. In this case, extra jets are still allowed, which maintains the full inclusiveness of the matched sample. 


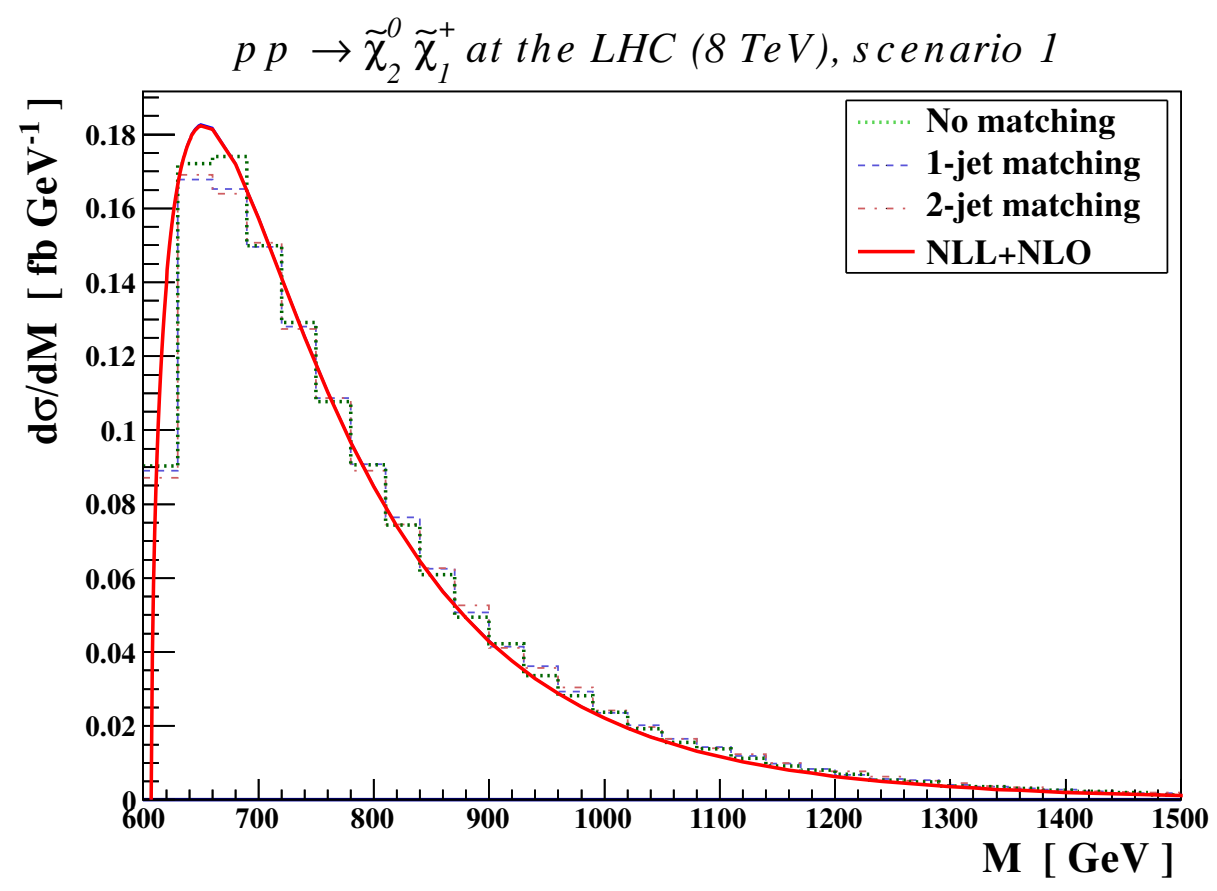

Figure 2. Distributions in the invariant mass $M$ of a $\tilde{\chi}_{2}^{0} \tilde{\chi}_{1}^{+}$pair with mass $304 \mathrm{GeV}$ each (benchmark point 1 ) at the LHC with $\sqrt{s}=8 \mathrm{TeV}$. We compare the NLO matched to the NLL (red, thick full) distribution to the results obtained after matching matrix elements containing no (green, dotted), one (blue, dashed), and up to two (red, dot-dashed) additional jets to parton showering.

In our simulation setup, we neglect all quark masses but the top mass and employ the leading order set of the MSTW 2008 parton density fits [46]. In contrast, the NLO and NLO+NLL results are based on the next-to-leading order set of the MSTW 2008 fits. We identify the factorization and renormalization scales as the average mass of the produced particles in the case of the three hard processes, i.e., without any extra parton as well as with one and with two additional (hard) radiations, while the renormalization scale used by the showering algorithm consists in the jet measure at each branching. The value of the total cross section associated to a matched event sample is usually rather close to the one of the original process, where no extra radiation is allowed, i.e., it is close to the value of the tree-level cross section. Therefore, we reweight the produced events uniformly, so that the total rate is now equal to the resummed cross sections given in the tables of section 2 . Let us note that we have also performed the on-shell subtraction of any intermediate resonance which could appear in the samples containing one or two extra partons using the narrow-width approximation, as those subprocesses are rather identified with other genuine processes such as the associated production of a gaugino and a squark or a gluino. Finally, the event samples have been analyzed with the program package MADANALYsis 5 [78]. 


\subsection{Distributions in the invariant mass of the gaugino pair}

In figure 2, we depict the invariant mass spectrum $\mathrm{d} \sigma / \mathrm{d} M$ for the associated production of a lightest chargino $\left(\tilde{\chi}_{1}^{+}\right)$and second lightest neutralino $\left(\tilde{\chi}_{2}^{0}\right)$ pair in the context of the benchmark scenario 1 presented in section 2.1. We compare the results obtained using the threshold resummation formalism (thick red full curve), found to be almost equivalent to those corresponding to a pure next-to-leading order calculation, to those generated by LO Monte Carlo simulations with MAdGrAPH and interfaced to the parton showering algorithm provided by PYTHIA. For the latter, we have normalized the total cross section to its resummed value of $40.51 \mathrm{fb}$ (see table 4 ). The spectrum starts at $M \sim 600 \mathrm{GeV}$, since the two gauginos are almost degenerate with a mass of about $300 \mathrm{GeV}$ each. The shape of the spectrum is then dictated by the nature of the produced superparticles, which are here mainly gaugino-like. This suppresses the $s$-channel $W$-boson exchange contributions with respect to $P$-wave production through $t$ - and $u$-channel squark exchange, the mass of the squarks being here of the order of $800 \mathrm{GeV}$. The resummed contributions increase the total rate in comparison to the pure next-to-leading order results only slightly, since threshold effects only become important when the invariant mass approaches the total center-of-mass energy.

The set of three curves obtained using the MADGRAPH and Pythia programs agree quite well with each other. They are related to three different ways which we have adopted to perform the matching procedure. The results represented by the green dotted curve have been obtained from tree-level matrix elements only, related hence to the process

$$
p p \rightarrow \tilde{\chi}_{2}^{0} \tilde{\chi}_{1}^{+}
$$

without any extra radiation. The parton-level events have then been passed to PYTHIA for parton showering, and no matching procedure has been applied. After accounting for a resummed $K$-factor normalizing the integrated distribution to $40.51 \mathrm{fb}$ (see table 4 ), one observes a good agreement with the resummed predictions. A similar behavior has already been observed in the context of $Z^{\prime}$ production at the LHC, where in ref. [33] resummed computations were confronted to results obtained with tree-level matrix-elements passed to a parton showering algorithm.

Applying the $k_{T}$-MLM matching procedure described in the beginning of section 3 , we compute matrix elements containing up to one additional QCD emission, i.e., considering the subprocesses

$$
p p \rightarrow \tilde{\chi}_{2}^{0} \tilde{\chi}_{1}^{+} \quad \text { and } \quad p p \rightarrow \tilde{\chi}_{2}^{0} \tilde{\chi}_{1}^{+} j
$$

where $j$ denotes a quark, an antiquark or a gluon, and match them consistently to parton showering. We obtain the results shown by the blue dashed curve of figure 2 after normalizing again the total cross section to its resummed value of $40.51 \mathrm{fb}$ (see table 4 ).

In a similar fashion, one can allow for matrix elements with up to two extra partons, i.e., we account for the processes

$$
p p \rightarrow \tilde{\chi}_{2}^{0} \tilde{\chi}_{1}^{+}, \quad p p \rightarrow \tilde{\chi}_{2}^{0} \tilde{\chi}_{1}^{+} j, \quad \text { and } \quad p p \rightarrow \tilde{\chi}_{2}^{0} \tilde{\chi}_{1}^{+} j j
$$




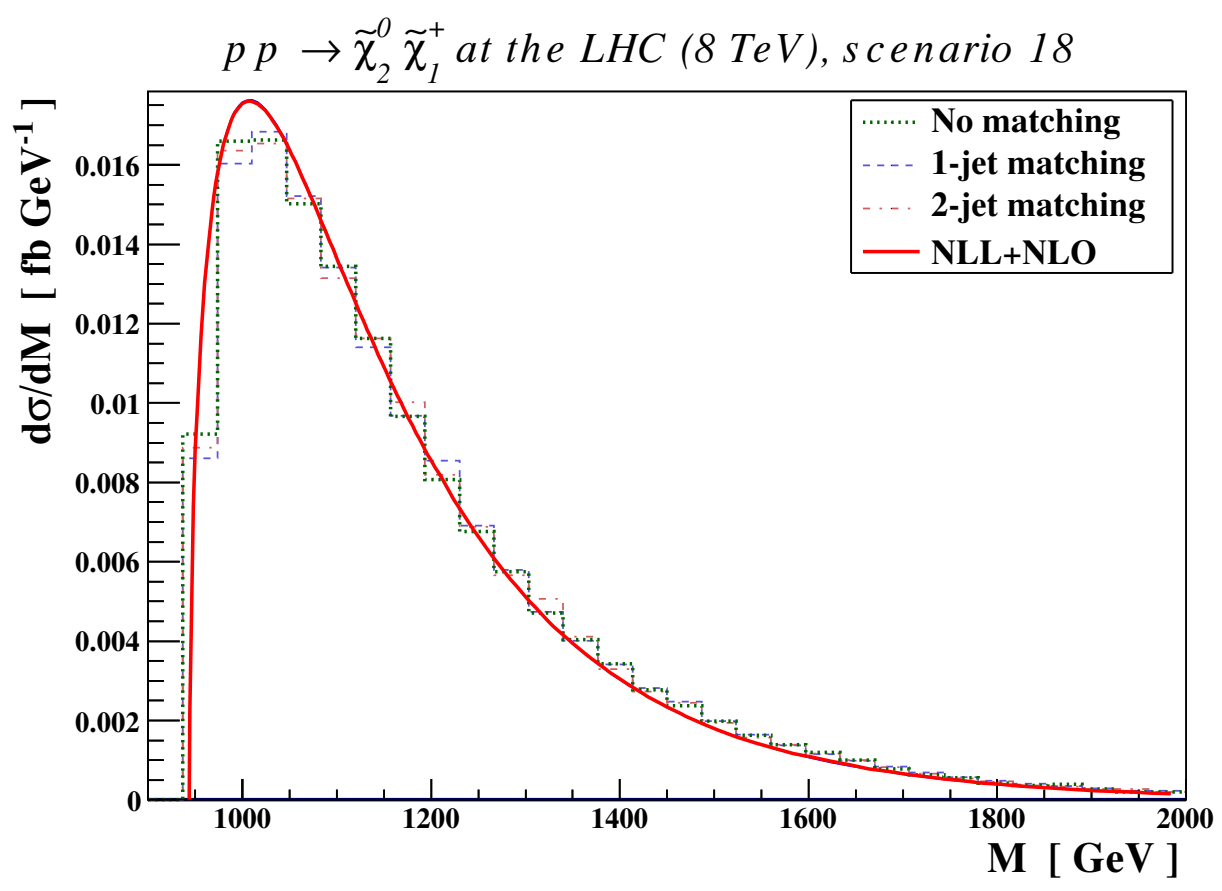

Figure 3. Same as figure 2 for the benchmark point 18. The gaugino masses are here $472 \mathrm{GeV}$. The distributions for the benchmark point 31 with gaugino masses of $479 \mathrm{GeV}$ are very similar and therefore not shown.

This leads to the dot-dashed red curve presented in figure 2. Again, since the matched total cross section is close to the unmatched one, the results have been normalized to $40.51 \mathrm{fb}$, including hence a resummed $K$-factor.

In the case of the benchmark scenarios 18 and 31, the gaugino masses are heavier, with $m_{\tilde{\chi}}=472 \mathrm{GeV}$ and $479 \mathrm{GeV}$, respectively. This reduces the total production cross section of an associated pair of the lightest chargino and the next-to-lightest neutralino by about an order of magnitude, which then reads $5.22 \mathrm{fb}$ for the scenario 18 (see table 5 ) and 4.81 $\mathrm{fb}$ for the scenario 31 (see table 6). Employing these values for the normalization of the results generated by MADGRAPH and PYTHIA, we confront them again to our NLL+NLO calculation for scenario 18 in figure 3 and for scenario 31 (not shown). One observes a similar behavior as for the scenario 1 shown in figure 2 .

\subsection{Distributions in the transverse momentum of the gaugino pair}

For scenario 1 at the LHC with a center-of-mass energy of $8 \mathrm{TeV}$, we show in figure 4 the transverse-momentum distribution $\mathrm{d} \sigma / \mathrm{d} p_{T}$ of a lightest chargino $\left(\tilde{\chi}_{1}^{+}\right)$and next-tolightest neutralino $\left(\tilde{\chi}_{2}^{0}\right)$ pair. As expected, the fixed order predictions at $\mathcal{O}\left(\alpha_{s}\right)$ (full blue curve) diverge as the transverse momentum $p_{T}$ tends to zero due to the unbalanced large logarithmic terms related to soft parton radiation. After their consistent matching to a resummation computation in the transverse-momentum regime at the next-to-leading logarithmic accuracy (thick red full curve), the results exhibit a finite behavior with a 

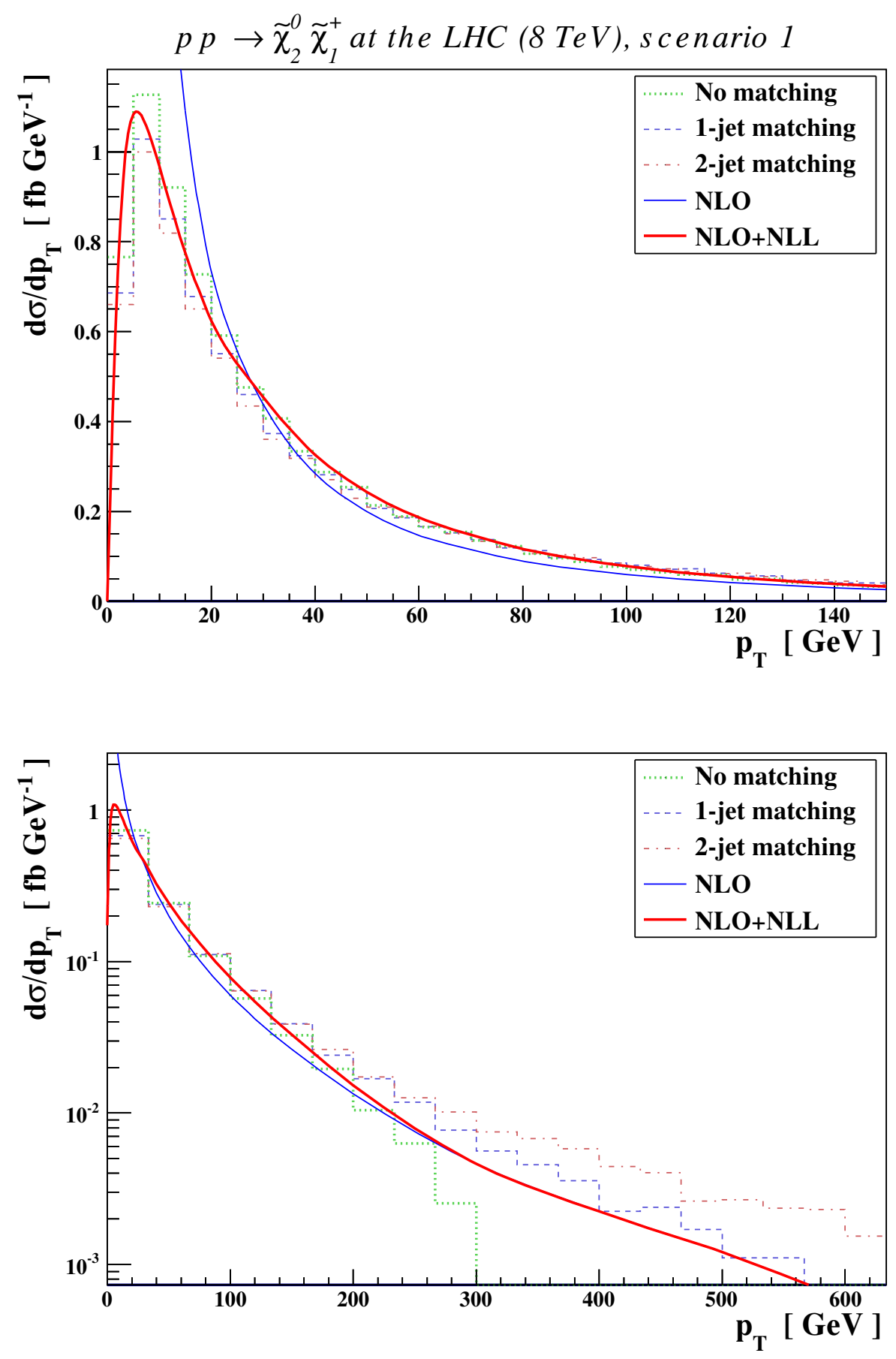

Figure 4. Distributions in the transverse momentum $p_{T}$ of a $\tilde{\chi}_{2}^{0} \tilde{\chi}_{1}^{+}$pair with mass $304 \mathrm{GeV}$ each (benchmark point 1) at the LHC with $\sqrt{s}=8 \mathrm{TeV}$. We compare fixed order at $\mathcal{O}\left(\alpha_{s}\right)($ blue, full) and NLL (red, thick full) distributions to the results obtained after matching matrix-elements containing no (green, dotted), one (blue, dashed), and up to two (red, dot-dashed) additional jets to parton showering in the small (top) and large (bottom) $p_{T}$ regions. 
maximum around a $p_{T}$-value of about $10 \mathrm{GeV}$. The effects of the resummation of the large logarithms extend to intermediate values of the transverse momentum, where the resummed predictions are considerably larger than those obtained from the pure fixed order computation as it has already been observed for slepton-pair production $[21,23]$ or other gaugino-pair production channels $[18,19]$.

We now turn to the confrontation of the resummed results to the three sets of predictions obtained after employing the MADGRAPH and PYTHIA generators, following the same approach as the one described in section 3.1. Again the total production rate associated to each of these three curves has been set to the value of the resummed total cross section of $40.51 \mathrm{fb}$ (see table 4 ). One observes very good agreement between the most precise distribution including a matching of the resummed results to the fixed order ones (thick red full curve) and the distributions obtained with MADGRAPH and Pythia after having matched the matrix elements containing one or two additional jets to parton showering (blue dashed and red dot-dashed curves in figure 4). Contrary, the simple application of a parton showering algorithm to the tree-level matrix element (green dotted curve on figure 4) leads to a slightly too soft spectrum as expected, since parton showering methods can not address properly the intermediate and large $p_{T}$ regions.

In contrast to the invariant-mass distributions, where the three Monte Carlo predictions (without matching, with a matching procedure of matrix elements accounting for at most one additional parton, and with a matching procedure of matrix elements containing at most two extra partons) have been found to be in a rather good agreement (see section 3.1), one can clearly here observe the effects of including additional partons when comparing the three distributions generated by the Monte Carlo simulators in figure 4. While the position of the peak does not depend much on the presence of additional partons at the matrix-element level, the global hardness of the spectrum does indeed highly depend on the contributions of these extra partons. One observes a very good agreement between the NLL+NLO results and the Monte Carlo predictions after matching parton showering to matrix elements containing up to one extra parton, which is not surprising since the same matrix elements, relevant for hard emissions, are included in the resummation computations. As resummation computations at NNLO+NNLL must still be performed, the accuracy of the Monte Carlo predictions with two hard jets matched to parton showers can at this point not yet be judged.

The same type of effects can be noticed for the benchmark scenario 18 in figure 5 and scenario 31 (not shown), the only difference being the overall scale of the distributions, which is about one order of magnitude smaller than in scenario 1 due to the larger gaugino masses.

In figures 6 and 7, we address the production of two other pairs of gauginos, i.e. of $\tilde{\chi}_{1}^{-} \tilde{\chi}_{2}^{0}$ and $\tilde{\chi}_{1}^{+} \tilde{\chi}_{1}^{-}$. As before, the Monte Carlo curves have been normalized according to the resummed results of table 4, i.e., to $17.05 \mathrm{fb}$ and $30.04 \mathrm{fb}$ for the associated production and the chargino pair production channels, respectively. The distributions are similar to those of the golden channel $\tilde{\chi}_{2}^{0} \tilde{\chi}_{1}^{+}$, but the absolute size of the latter (see figure 4) exceeds the one of the $\tilde{\chi}_{1}^{-} \tilde{\chi}_{2}^{0}$ channel (see figure 6 ) as expected for proton-proton collisions where the production of a positively charged final state is favored with respect to a negatively charged final state. 

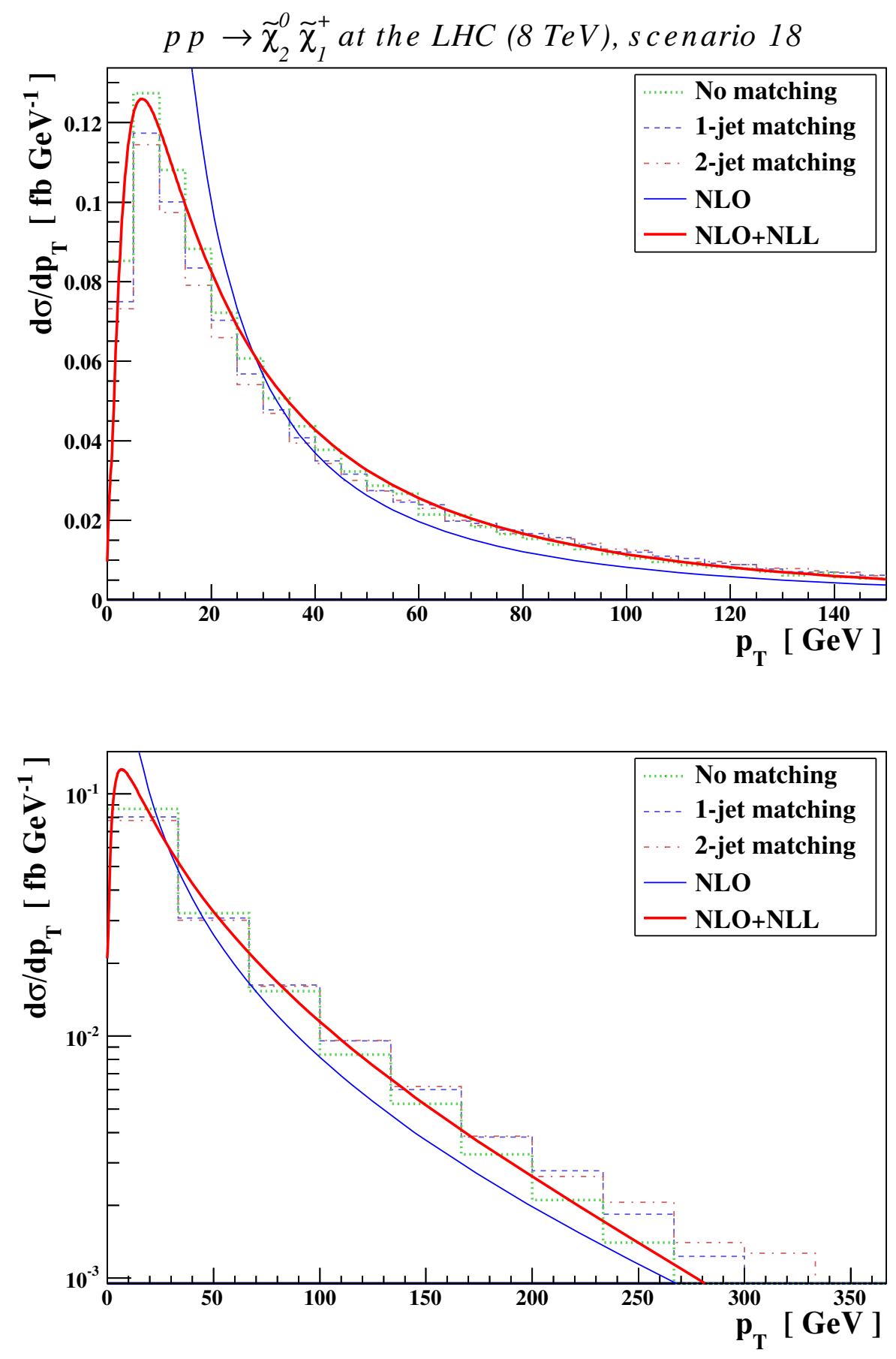

Figure 5. Same as figure 4 for the benchmark point 18. The gaugino masses are here $472 \mathrm{GeV}$. The distributions for the benchmark point 31 with gaugino masses of $479 \mathrm{GeV}$ are very similar and therefore not shown. 

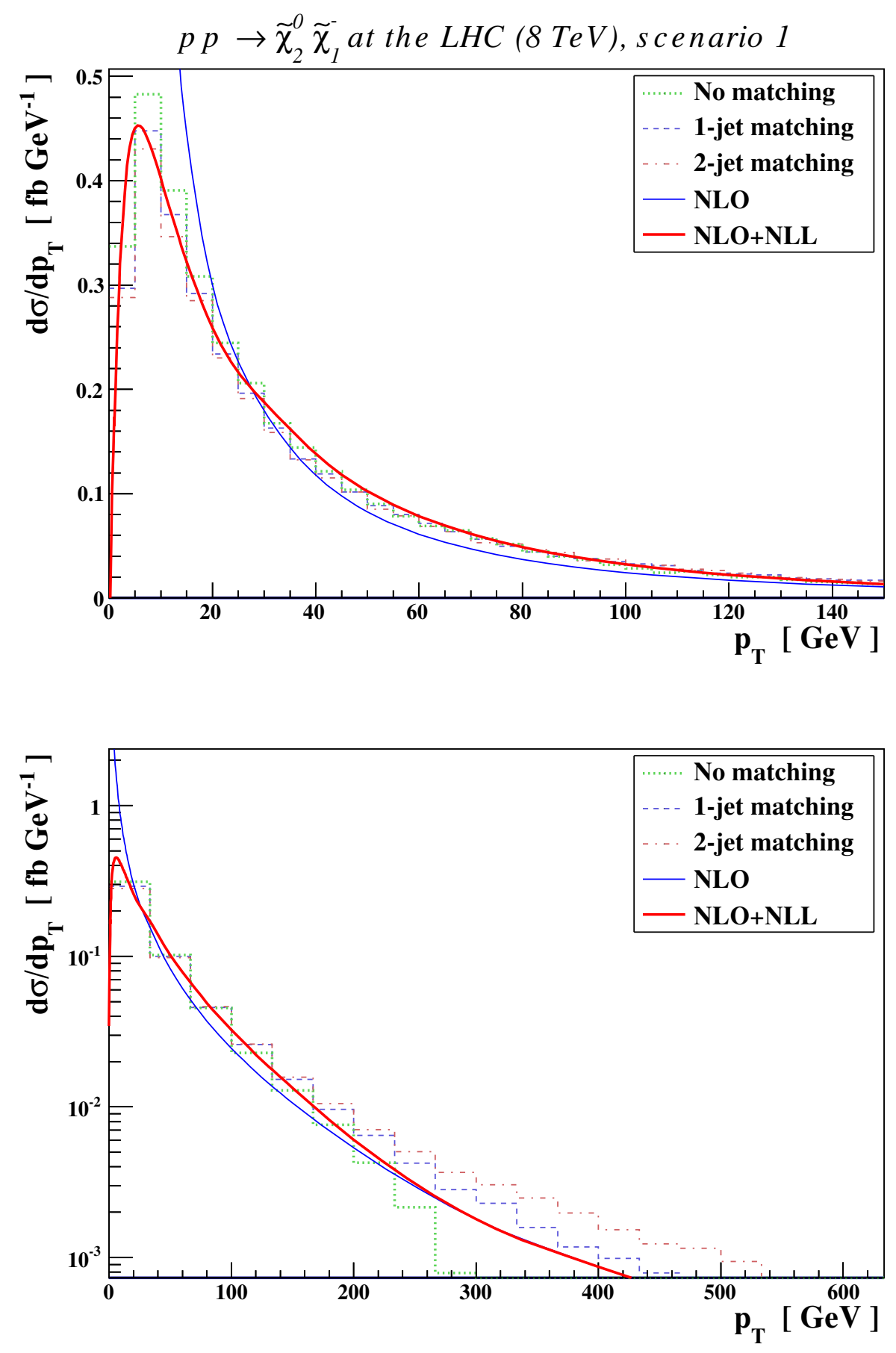

Figure 6. Same as figure 4 for the production of a $\tilde{\chi}_{1}^{-} \tilde{\chi}_{2}^{0}$ associated pair.

\section{Conclusion}

With a Higgs boson of about $125 \mathrm{GeV}$ to be unveiled, but no hint yet for any colored supersymmetric particle, experimental searches are now more and more focusing on the pair production of the superpartners of the gauge and Higgs bosons. 

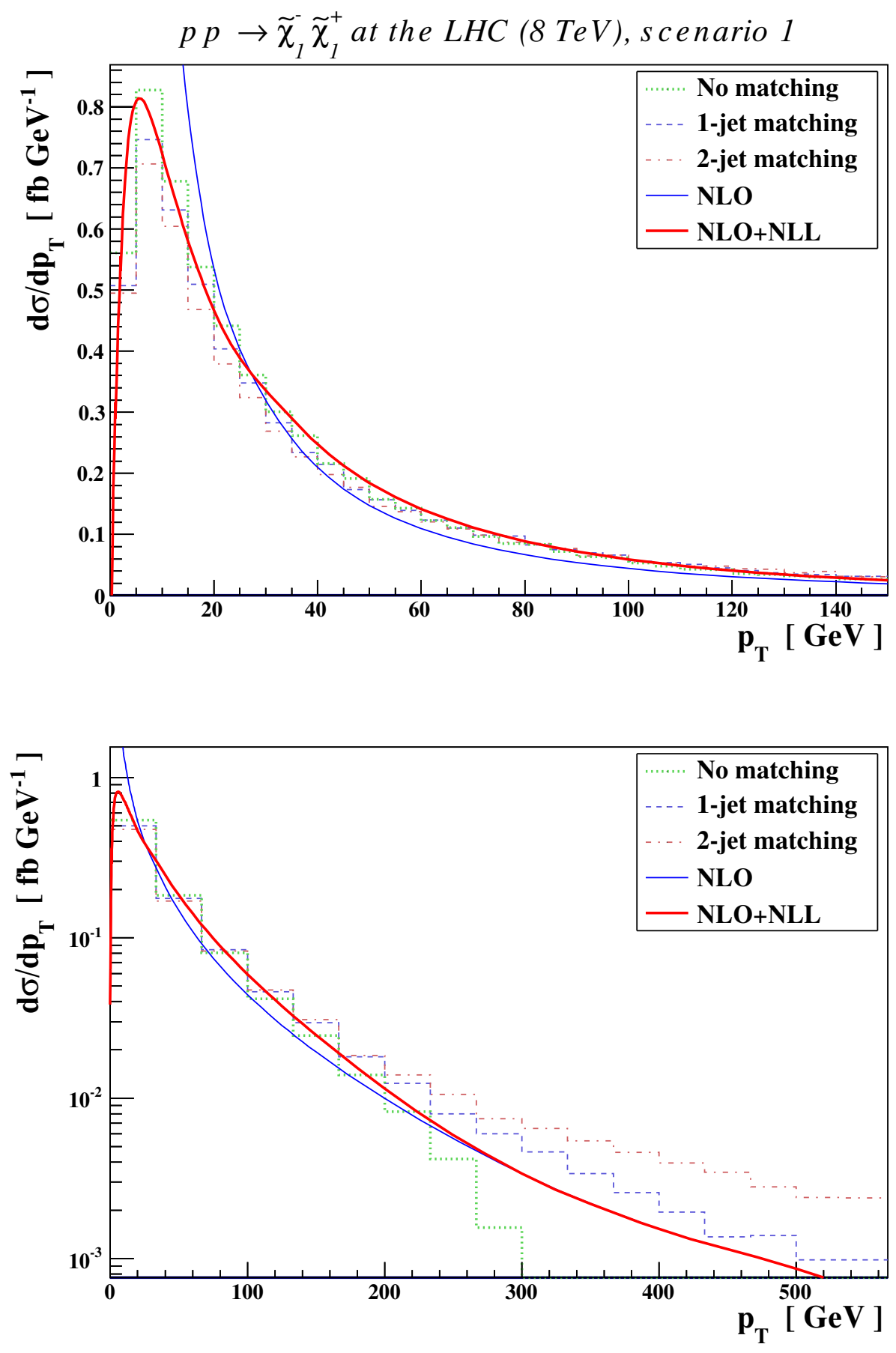

Figure 7. Same as figure 4 for the production of a $\tilde{\chi}_{1}^{+} \tilde{\chi}_{1}^{-}$pair.

In this paper, we have updated the total cross sections related to the production of various gaugino pairs at the LHC, running presently at a center-of-mass energy of $8 \mathrm{TeV}$. We have presented results at the leading order and the next-to-leading order of perturbative QCD and matched, in addition, the NLO results to threshold resummation at the next- 
to-leading logarithmic accuracy in order to consistently account for the large logarithmic corrections arising from soft parton emission. Our cross sections have been given for several benchmark scenarios motivated by the recent LHC supersymmetric searches, together with the theoretical uncertainties stemming from scale variation and the choice of the parton density sets. We have shown that resummation at the NLL accuracy allows for a drastic reduction of the scale dependence of the cross section to less or about a percent, in contrast to the PDF errors which are now the dominant source of theoretical uncertainties and in general of the order of 2-3 percent.

We have also analyzed the precision of the traditional experimental approach for supersymmetric process simulation consisting in merging parton showering with multi-parton matrix elements. We have shown that the predictions obtained following this approach are largely in agreement with the most precise theoretical computations matching resummation at the NLL level and fixed order calculations at the NLO accuracy.

We finally emphasize that even if increasing experimental limits on the squark and gluino masses might exclude the investigated scenarios, the (lower) gaugino masses may still remain allowed so that our results could stay valid in the context of more general SUSY-breaking scenarios yielding gaugino masses of the same order. As already stated above, further numerical results are available from the authors upon request.

\section{A Total cross sections at $\sqrt{s}=7 \mathrm{TeV}$ for benchmark points 1,18 , and 31}

In this appendix, we present in tables 7,8 , and 9 the total production cross sections for the same combinations of neutralino and chargino pairs in the context of the benchmark points 1, 18 and 31 as in section 2.2, but now for data taken earlier at the LHC at a center-of-mass energy of $\sqrt{s}=7 \mathrm{TeV}$. We give again numerical results at the leading and next-to-leading order of perturbative QCD as well as after matching the NLO results to threshold resummation.

Due to the slightly lower available energy, the cross sections are smaller by about $30 \%$ with respect to those presented in tables 4-6. In particular, the cross sections for heavy gaugino pairs do not exceed $0.1 \mathrm{fb}$ at the benchmark points 18 and 31, so that they are no longer listed. On the other hand, the ATLAS and CMS experiments accumulated in 2011 an integrated luminosity of around $6 \mathrm{fb}^{-1}$ each, so that for the light channels a significant number of gaugino events could have been produced at all three benchmark points considered here.

\section{Acknowledgments}

The authors are grateful to Johan Alwall and Olivier Mattelaer for their help with the MADGRAPH program. This work has been supported by the BMBF Theorie-Verbund and by the Theory-LHC-France initiative of the CNRS/IN2P3. 


\begin{tabular}{|c|c|c|c|c|c|}
\hline Process & $m_{1}[\mathrm{GeV}]$ & $m_{2}[\mathrm{GeV}]$ & $\mathrm{LO}[\mathrm{fb}]$ & NLO $[\mathrm{fb}]$ & $\mathrm{NLO}+\mathrm{NLL}[\mathrm{fb}]$ \\
\hline$p p \rightarrow \chi_{1}^{0} \chi_{1}^{0}$ & 161.7 & 161.7 & $0.61_{-6.1 \%}^{+6.7 \%}$ & $0.79_{-3.2 \%-2.1 \%}^{+3.7 \%+3.0 \%}$ & $0.77_{-0.7 \%-2.1 \%}^{+0.4 \%+2.9 \%}$ \\
\hline$p p \rightarrow \chi_{1}^{0} \chi_{1}^{-}$ & 161.7 & 303.5 & $0.12_{-6.3 \%}^{+7.0 \%}$ & $0.15_{-2.6 \%-2.5 \%}^{+2.6 \%+3.1 \%}$ & $0.14_{-0.4 \%-2.7 \%}^{+0.0 \%+3.1 \%}$ \\
\hline$p p \rightarrow \chi_{2}^{0} \chi_{2}^{0}$ & 303.8 & 303.8 & $0.58_{-8.7 \%}^{+10.2 \%}$ & $0.73_{-3.8 \%-2.2 \%}^{+3.7 \%+3.5 \%}$ & $0.71_{-0.1 \%-2.1 \%}^{+0.1 \%+3.7 \%}$ \\
\hline$p p \rightarrow \chi_{2}^{0} \chi_{3}^{0}$ & 303.8 & 526.5 & $0.14_{-9.0 \%}^{+10.5 \%}$ & $0.17_{-3.2 \%-2.4 \%}^{+2.8 \%+3.6 \%}$ & $0.17_{-0.5 \%-2.4 \%}^{+0.2 \%+3.7 \%}$ \\
\hline$p p \rightarrow \chi_{2}^{0} \chi_{1}^{-}$ & 303.8 & 303.5 & $10.42_{-6.9 \%}^{+7.7 \%}$ & $12.33_{-2.0 \%-2.7 \%}^{+1.7 \%+3.3 \%}$ & $\begin{array}{l}12.18_{-0.8 \%-2.7 \%}^{+0.3 \%+3.3 \%} \\
\end{array}$ \\
\hline$p p \rightarrow \chi_{3}^{0} \chi_{4}^{0}$ & 526.5 & 542.4 & $0.52_{-10.2 \%}^{+12.2 \%}$ & $0.60_{-3.6 \%-2.6 \%}^{+3.1 \%+4.4 \%}$ & $0.59_{-1.2 \%-2.8 \%}^{+0.6 \%+4.5 \%}$ \\
\hline$p p \rightarrow \chi_{3}^{0} \chi_{2}^{-}$ & 526.5 & 542.2 & $0.25_{-10.4 \%}^{+12.4 \%}$ & $0.30_{-3.6 \%-4.2 \%}^{+3.1 \%+5.7 \%}$ & $0.30_{-1.3 \%-4.1 \%}^{+0.7 \%+5.8 \%}$ \\
\hline$p p \rightarrow \chi_{4}^{0} \chi_{2}^{-}$ & 542.4 & 542.2 & $0.24_{-10.4 \%}^{+12.5 \%}$ & $\begin{array}{r}0.28_{-3.5 \%-4.3 \%}^{+2.9 \%+5.8 \%} \\
\end{array}$ & $0.28_{-1.8 \%}^{+0.8 \%+5.9 \%}$ \\
\hline$p p \rightarrow \chi_{1}^{+} \chi_{1}^{0}$ & 303.5 & 161.7 & $0.29_{-6.2 \%}^{+6.9 \%}$ & $\begin{array}{l}0.35_{-2.6 \%}^{+2.6 \%}+3.0 \% \\
\end{array}$ & $0.35_{-0.4 \%-2.2 \%}^{+0.1 \%+3.0 \%}$ \\
\hline$p p \rightarrow \chi_{1}^{+} \chi_{2}^{0}$ & 303.5 & 303.8 & $26.71_{-6.6 \%}^{+7.4 \%}$ & $30.78_{-1.9 \%-2.3 \%}^{+1.7 \%+3.2 \%}$ & $30.47_{-0.4 \%-2.3 \%}^{+0.0 \%+3.2 \%}$ \\
\hline$p p \rightarrow \chi_{1}^{+} \chi_{3}^{0}$ & 303.5 & 526.5 & $0.24_{-8.8 \%}^{+10.4 \%}$ & $0.28_{-3.2 \%-2.6 \%}^{+2.8 \%+4.0 \%}$ & $0.27_{-0.3 \%-2.6 \%}^{+0.0 \%+4.1 \%}$ \\
\hline$p p \rightarrow \chi_{1}^{+} \chi_{1}^{-}$ & 303.5 & 303.5 & $18.90_{-6.8 \%}^{+7.6 \%}$ & $22.18_{-2.1 \%-2.1 \%}^{+1.8 \%+2.9 \%}$ & $21.93_{-0.6 \%-2.1 \%}^{+0.1 \%+2.9 \%}$ \\
\hline$p p \rightarrow \chi_{2}^{+} \chi_{3}^{0}$ & 542.2 & 526.5 & $0.81_{-10.4 \%}^{+12.4 \%}$ & $0.91_{-3.7 \%-3.0 \%}^{+3.1 \%+4.9 \%}$ & $0.91_{-1.0 \%-3.0 \%}^{+0.5 \%+4.8 \%}$ \\
\hline$p p \rightarrow \chi_{2}^{+} \chi_{4}^{0}$ & 542.2 & 542.4 & $0.76_{-10.4 \%}^{+12.5 \%}$ & $0.85_{-3.6 \%-3.0 \%}^{+3.0 \%+4.9 \%}$ & $0.85_{-1.1 \%-3.3 \%}^{+0.6 \%+4.9 \%}$ \\
\hline$p p \rightarrow \chi_{2}^{+} \chi_{2}^{-}$ & 542.2 & 542.2 & $0.54_{-10.1 \%}^{+12.1 \%}$ & $\begin{array}{l}0.62_{-3.5 \%-2.7 \%}^{+2.9 \%+4.6 \%} \\
\end{array}$ & $0.61_{-1.2 \%-2.7 \%}^{+0.6 \%+4.6 \%}$ \\
\hline
\end{tabular}

Table 7. Total cross sections related to the production of various gaugino pairs of masses $m_{1}$ and $m_{2}$, presented together with the associated scale and PDF uncertainties for the LHC running at a center-of-mass energy of $\sqrt{s}=7 \mathrm{TeV}$ in the context of the benchmark point 1 of the LPCC numbering scheme. The cross sections are given at the leading order and next-to-leading order of perturbative QCD and matched to threshold resummation. The PDF uncertainties are not shown for the LO results. Any cross section smaller than $0.1 \mathrm{fb}$ is omitted.

\begin{tabular}{|l|l|l|l|l|l|}
\hline Process & $m_{1}[\mathrm{GeV}]$ & $m_{2}[\mathrm{GeV}]$ & LO [fb] & NLO [fb] & NLO+NLL [fb] \\
\hline$p p \rightarrow \chi_{2}^{0} \chi_{1}^{-}$ & 471.9 & 471.8 & $1.05_{-9.4 \%}^{+11.1 \%}$ & $1.21_{-2.7 \%}^{+2.0 \%+4.7 \%}$ & $1.19_{-1.4 \%-3.4 \%}^{+0.8 \%+4.8 \%}$ \\
$p p \rightarrow \chi_{1}^{+} \chi_{2}^{0}$ & 471.8 & 471.9 & $3.20_{-9.3 \%}^{+11.0 \%}$ & $3.53_{-2.7 \%}^{+2.0 \%}+. .3 \%$ & $3.49_{-1.7 \%}^{+0.6 \%+4.4 \%}$ \\
$p p \rightarrow \chi_{1}^{+} \chi_{1}^{-}$ & 471.8 & 471.8 & $2.07_{-9.3 \%}^{+11.0 \%}$ & $2.33_{-2.8 \%-2.4 \%}^{+2.1 \%}+3.9 \%$ & $2.30_{-1.3 \%-2.5 \%}^{+0.6 \%+3.8 \%}$ \\
\hline
\end{tabular}

Table 8. Same as table 7 for the benchmark point 18 of the LPCC numbering scheme.

\begin{tabular}{|c|c|c|c|c|c|}
\hline Process & $m_{1}[\mathrm{GeV}]$ & $m_{2}[\mathrm{GeV}]$ & LO [fb] & NLO [fb] & $\mathrm{NLO}+\mathrm{NLL}[\mathrm{fb}]$ \\
\hline$p p \rightarrow \chi_{2}^{0} \chi_{1}^{-}$ & 478.5 & 478.5 & $0.96_{-9.5 \%}^{+11.2 \%}$ & $\begin{array}{l}1.10_{-2.7 \%}^{+2.0 \%+4.8 \%} \\
-3.5 \%\end{array}$ & $1.08_{-1.5 \%-3.6 \%}^{+0.9 \%+4.6 \%}$ \\
\hline$p p \rightarrow \chi_{1}^{+} \chi_{2}^{0}$ & 478.5 & 478.5 & $2.94_{-9.4 \%}^{+11.1 \%}$ & $3.23_{-2.0 \%-2.4 \%}^{+2.0 \%+4.4 \%}$ & $3.20_{-1.4 \%-2.6 \%}^{+0.4 \%+4.6 \%}$ \\
\hline$p p \rightarrow \chi_{1}^{+} \chi_{1}^{-}$ & 478.5 & 478.5 & $1.90_{-9.4 \%}^{+11.1 \%}$ & $2.13_{-2.8 \%-2.4 \%}^{+2.1 \%+3.9 \%}$ & $2.11_{-1.1 \%-2.6 \%}^{+0.5 \%+3.8 \%}$ \\
\hline
\end{tabular}

Table 9. Same as table 7 for the benchmark point 31 of the LPCC numbering scheme.

Open Access. This article is distributed under the terms of the Creative Commons Attribution License which permits any use, distribution and reproduction in any medium, provided the original author(s) and source are credited. 


\section{References}

[1] ATLAS collaboration, G. Aad et al., Combined search for the standard model Higgs boson using up to $4.9 \mathrm{fb}^{-1}$ of pp collision data at $\sqrt{\mathrm{s}}=7 \mathrm{TeV}$ with the ATLAS detector at the LHC, Phys. Lett. B 710 (2012) 49 [arXiv:1202.1408] [InSPIRE].

[2] CMS collaboration, S. Chatrchyan et al., Combined results of searches for the standard model Higgs boson in pp collisions at $\sqrt{s}=7$ TeV, Phys. Lett. B 710 (2012) 26 [arXiv:1202.1488] [INSPIRE].

[3] H.P. Nilles, Supersymmetry, supergravity and particle physics, Phys. Rept. 110 (1984) 1 [INSPIRE].

[4] H.E. Haber and G.L. Kane, The search for supersymmetry: probing physics beyond the standard model, Phys. Rept. 117 (1985) 75 [INSPIRE].

[5] CMS collaborations collaboration, S. Lowette and o.b. o.t. ATLAS, Supersymmetry searches with ATLAS and CMS, arXiv:1205.4053 [INSPIRE].

[6] S. AbdusSalam, B. Allanach, H. Dreiner, J. Ellis, U. Ellwanger, et al., Benchmark models, planes, lines and points for future SUSY searches at the LHC,

Eur. Phys. J. C 71 (2011) 1835 [arXiv:1109.3859] [InSPIRE].

[7] A. Kulesza and L. Motyka, Threshold resummation for squark-antisquark and gluino-pair production at the LHC, Phys. Rev. Lett. 102 (2009) 111802 [arXiv:0807.2405] [INSPIRE].

[8] A. Kulesza and L. Motyka, Soft gluon resummation for the production of gluino-gluino and squark-antisquark pairs at the LHC, Phys. Rev. D 80 (2009) 095004 [arXiv:0905.4749] [INSPIRE].

[9] W. Beenakker, S. Brensing, M. Krämer, A. Kulesza, E. Laenen and I. Niessen, Soft-gluon resummation for squark and gluino hadroproduction, JHEP 12 (2009) 041 [arXiv:0909.4418] [INSPIRE].

[10] W. Beenakker, S. Brensing, M. Krämer, A. Kulesza, E. Laenen and I. Niessen, Supersymmetric top and bottom squark production at hadron colliders, JHEP 08 (2010) 098 [arXiv: 1006.4771] [INSPIRE].

[11] W. Beenakker, S. Brensing, M. Krämer, A. Kulesza, E. Laenen, et al., Squark and gluino hadroproduction, Int. J. Mod. Phys. A 26 (2011) 2637 [arXiv:1105.1110] [InSPIRE].

[12] M. Krämer, A. Kulesza, R. van der Leeuw, M. Mangano, S. Padhi, et al., Supersymmetry production cross sections in pp collisions at $\sqrt{s}=7 \mathrm{Te} V$, arXiv:1206.2892 [INSPIRE].

[13] ATLAS collaboration, G. Aad et al., Search for supersymmetry in events with three leptons and missing transverse momentum in $\sqrt{s}=7 \mathrm{TeV}$ pp collisions with the ATLAS detector, Phys. Rev. Lett. 108 (2012) 261804 [arXiv:1204.5638] [INSPIRE].

[14] D. Curtin, P. Jaiswal and P. Meade, Charginos hiding in plain sight, arXiv: 1206.6888 [INSPIRE].

[15] J. Debove, B. Fuks and M. Klasen, Model-independent analysis of gaugino-pair production in polarized and unpolarized hadron collisions, Phys. Rev. D 78 (2008) 074020 [arXiv: 0804.0423] [INSPIRE].

[16] J. Debove, B. Fuks and M. Klasen, Threshold resummation for gaugino pair production at hadron colliders, Nucl. Phys. B 842 (2011) 51 [arXiv:1005.2909] [INSPIRE]. 
[17] W. Beenakker, M. Klasen, M. Krämer, T. Plehn, M. Spira, et al., The production of charginos/neutralinos and sleptons at hadron colliders, Phys. Rev. Lett. 83 (1999) 3780 [Erratum ibid. 100 (2008) 029901] [hep-ph/9906298] [INSPIRE].

[18] J. Debove, B. Fuks and M. Klasen, Transverse-momentum resummation for gaugino-pair production at hadron colliders, Phys. Lett. B 688 (2010) 208 [arXiv:0907.1105] [INSPIRE].

[19] J. Debove, B. Fuks and M. Klasen, Joint resummation for gaugino pair production at hadron colliders, Nucl. Phys. B 849 (2011) 64 [arXiv:1102.4422] [InSPIRE].

[20] G. Bozzi, B. Fuks and M. Klasen, Slepton production in polarized hadron collisions, Phys. Lett. B 609 (2005) 339 [hep-ph/0411318] [INSPIRE].

[21] G. Bozzi, B. Fuks and M. Klasen, Transverse-momentum resummation for slepton-pair production at the CERN LHC, Phys. Rev. D 74 (2006) 015001 [hep-ph/0603074] [INSPIRE].

[22] G. Bozzi, B. Fuks and M. Klasen, Threshold resummation for slepton-pair production at hadron colliders, Nucl. Phys. B 777 (2007) 157 [hep-ph/0701202] [INSPIRE].

[23] G. Bozzi, B. Fuks and M. Klasen, Joint resummation for slepton pair production at hadron colliders, Nucl. Phys. B 794 (2008) 46 [arXiv:0709.3057] [InSPIRE].

[24] T. Plehn, Measuring the MSSM Lagrangean, Czech. J. Phys. 55 (2005) B213 [hep-ph/0410063] [INSPIRE].

[25] T. Binoth, D. Goncalves Netto, D. Lopez-Val, K. Mawatari, T. Plehn and I. Wigmore, Automized squark-neutralino production to next-to-leading order,

Phys. Rev. D 84 (2011) 075005 [arXiv: 1108.1250] [INSPIRE].

[26] E.L. Berger, M. Klasen and T.M. Tait, Associated production of gauginos and gluinos at hadron colliders in next-to-leading order SUSY QCD, Phys. Lett. B 459 (1999) 165 [hep-ph/9902350] [INSPIRE].

[27] E.L. Berger, M. Klasen and T.M. Tait, Next-to-leading order SUSY QCD predictions for associated production of gauginos and gluinos, Phys. Rev. D 62 (2000) 095014 [Erratum-ibid. 67 (2003) 099901] [hep-ph/0005196] [INSPIRE].

[28] M. Spira, Higgs and SUSY particle production at hadron colliders, hep-ph/0211145 [INSPIRE].

[29] G. Corcella, I. Knowles, G. Marchesini, S. Moretti, K. Odagiri, et al., HERWIG 6: an event generator for hadron emission reactions with interfering gluons (including supersymmetric processes), JHEP 01 (2001) 010 [hep-ph/0011363] [INSPIRE].

[30] T. Sjöstrand, S. Mrenna and P.Z. Skands, PYTHIA 6.4 physics and manual, JHEP 05 (2006) 026 [hep-ph/0603175] [INSPIRE].

[31] M.L. Mangano, M. Moretti, F. Piccinini, R. Pittau and A.D. Polosa, ALPGEN, a generator for hard multiparton processes in hadronic collisions, JHEP 07 (2003) 001 [hep-ph/0206293] [INSPIRE].

[32] J. Alwall, M. Herquet, F. Maltoni, O. Mattelaer and T. Stelzer, MadGraph 5 : going beyond, JHEP 06 (2011) 128 [arXiv:1106.0522] [INSPIRE].

[33] B. Fuks, M. Klasen, F. Ledroit, Q. Li and J. Morel, Precision predictions for Z' - Production at the CERN LHC: QCD matrix elements, parton showers and joint resummation, Nucl. Phys. B 797 (2008) 322 [arXiv:0711.0749] [INSPIRE]. 
[34] C. Weydert, S. Frixione, M. Herquet, M. Klasen, E. Laenen, et al., Charged Higgs boson production in association with a top quark in MC@NLO, Eur. Phys. J. C 67 (2010) 617 [arXiv: 0912.3430] [INSPIRE].

[35] M. Klasen, K. Kovarik, P. Nason and C. Weydert, Associated production of charged Higgs bosons and top quarks with POWHEG, arXiv:1203.1341 [INSPIRE].

[36] B. Allanach, M. Battaglia, G. Blair, M.S. Carena, A. De Roeck, et al., The snowmass points and slopes: benchmarks for SUSY searches, Eur. Phys. J. C 25 (2002) 113 [hep-ph/0202233] [INSPIRE].

[37] J.A. Aguilar-Saavedra, A. Ali, B.C. Allanach, R.L. Arnowitt, H.A. Baer, et al., Supersymmetry parameter analysis: SPA convention and project, Eur. Phys. J. C 46 (2006) 43 [hep-ph/0511344] [INSPIRE].

[38] CERN, LHC physics center at CERN, http://cern.ch/lpcc.

[39] A. Kulesza et al., LHC SUSY cross section working group, https://twiki.cern.ch/twiki/bin/view/LHCPhysics/SUSYCrossSections.

[40] V.D. Barger, R. Robinett, W.-Y. Keung and R. Phillips, Production of gauge fermions at colliders, Phys. Lett. B 131 (1983) 372 [INSPIRE].

[41] S. Dawson, E. Eichten and C. Quigg, Search for supersymmetric particles in hadron - Hadron collisions, Phys. Rev. D 31 (1985) 1581 [INSPIRE].

[42] M. Klasen, Determining SUSY particle mixing with polarized hadron beams, PoS DIS2010 (2010) 233 [arXiv: 1005.3503] [INSPIRE].

[43] G. Bozzi, B. Fuks, B. Herrmann and M. Klasen, Squark and gaugino hadroproduction and decays in non-minimal flavour violating supersymmetry, Nucl. Phys. B 787 (2007) 1 [arXiv: 0704.1826] [INSPIRE].

[44] F. del Aguila, J. Aguilar-Saavedra, B. Allanach, J. Alwall, Y. Andreev, et al., Collider aspects of flavour physics at high Q, Eur. Phys. J. C 57 (2008) 183 [arXiv:0801.1800] [InSPIRE].

[45] B. Fuks, B. Herrmann and M. Klasen, Flavour violation in gauge-mediated supersymmetry breaking models: experimental constraints and phenomenology at the $\mathrm{LHC}$, Nucl. Phys. B $\mathbf{8 1 0}$ (2009) 266 [arXiv:0808.1104] [InSPIRE].

[46] A. Martin, W. Stirling, R. Thorne and G. Watt, Parton distributions for the LHC, Eur. Phys. J. C 63 (2009) 189 [arXiv:0901.0002] [inSPIRE].

[47] CDF AND D0 collaboration, T.E.W. Group, Combination of CDF and D0 results on the mass of the top quark, arXiv:0903.2503 [INSPIRE].

[48] Particle Data Group collaboration, K. Nakamura et al., Review of particle physics, J. Phys. G 37 (2010) 075021 [inSPIRE].

[49] A. Djouadi, J.-L. Kneur and G. Moultaka, Suspect: a Fortran code for the supersymmetric and Higgs particle spectrum in the MSSM, Comput. Phys. Commun. 176 (2007) 426 [hep-ph/0211331] [INSPIRE].

[50] A. Vogt, Next-to-next-to-leading logarithmic threshold resummation for deep inelastic scattering and the Drell-Yan process, Phys. Lett. B 497 (2001) 228 [hep-ph/0010146] [INSPIRE].

[51] M. Krämer, E. Laenen and M. Spira, Soft gluon radiation in Higgs boson production at the LHC, Nucl. Phys. B 511 (1998) 523 [hep-ph/9611272] [INSPIRE]. 
[52] S. Catani, D. de Florian and M. Grazzini, Higgs production in hadron collisions: soft and virtual QCD corrections at NNLO, JHEP 05 (2001) 025 [hep-ph/0102227] [INSPIRE].

[53] A. Kulesza, G.F. Sterman and W. Vogelsang, Joint resummation in electroweak boson production, Phys. Rev. D 66 (2002) 014011 [hep-ph/0202251] [INSPIRE].

[54] L.G. Almeida, G.F. Sterman and W. Vogelsang, Threshold resummation for di-hadron production in hadronic collisions, Phys. Rev. D 80 (2009) 074016 [arXiv:0907.1234] [INSPIRE].

[55] H. Contopanagos and G.F. Sterman, Principal value resummation, Nucl. Phys. B 419 (1994) 77 [hep-ph/9310313] [INSPIRE].

[56] S. Catani, M.L. Mangano, P. Nason and L. Trentadue, The resummation of soft gluons in hadronic collisions, Nucl. Phys. B 478 (1996) 273 [hep-ph/9604351] [INSPIRE].

[57] M. Bahr, S. Gieseke, M. Gigg, D. Grellscheid, K. Hamilton, et al., HERWIG++ physics and manual, Eur. Phys. J. C 58 (2008) 639 [arXiv:0803.0883] [INSPIRE].

[58] T. Sjöstrand, S. Mrenna and P.Z. Skands, A brief introduction to PYTHIA 8.1, Comput. Phys. Commun. 178 (2008) 852 [arXiv:0710.3820] [InSPIRE].

[59] S. Frixione and B.R. Webber, Matching NLO QCD computations and parton shower simulations, JHEP 06 (2002) 029 [hep-ph/0204244] [INSPIRE].

[60] S. Frixione, P. Nason and B.R. Webber, Matching NLO QCD and parton showers in heavy flavor production, JHEP 08 (2003) 007 [hep-ph/0305252] [INSPIRE].

[61] P. Nason, A new method for combining NLO QCD with shower Monte Carlo algorithms, JHEP 11 (2004) 040 [hep-ph/0409146] [INSPIRE].

[62] S. Catani, F. Krauss, R. Kuhn and B. Webber, QCD matrix elements + parton showers, JHEP 11 (2001) 063 [hep-ph/0109231] [INSPIRE].

[63] F. Krauss, Matrix elements and parton showers in hadronic interactions, JHEP 08 (2002) 015 [hep-ph/0205283] [INSPIRE].

[64] M.L. Mangano, M. Moretti, F. Piccinini and M. Treccani, Matching matrix elements and shower evolution for top-quark production in hadronic collisions, JHEP 01 (2007) 013 [hep-ph/0611129] [INSPIRE].

[65] S. Mrenna and P. Richardson, Matching matrix elements and parton showers with HERWIG and PYTHIA, JHEP 05 (2004) 040 [hep-ph/0312274] [INSPIRE].

[66] F. Krauss, A. Schalicke, S. Schumann and G. Soff, Simulating $W / Z+$ jets production at the Tevatron, Phys. Rev. D 70 (2004) 114009 [hep-ph/0409106] [InSPIRE].

[67] S. Hoeche, F. Krauss, N. Lavesson, L. Lönnblad, M. Mangano, et al., Matching parton showers and matrix elements, hep-ph/0602031 [INSPIRE].

[68] J. Alwall, S. Hoche, F. Krauss, N. Lavesson, L. Lönnblad, et al., Comparative study of various algorithms for the merging of parton showers and matrix elements in hadronic collisions, Eur. Phys. J. C 53 (2008) 473 [arXiv:0706.2569] [InSPIRE].

[69] J. Alwall, S. de Visscher and F. Maltoni, QCD radiation in the production of heavy colored particles at the LHC, JHEP 02 (2009) 017 [arXiv:0810.5350] [INSPIRE].

[70] P. de Aquino, K. Hagiwara, Q. Li and F. Maltoni, Simulating graviton production at hadron colliders, JHEP 06 (2011) 132 [arXiv:1101.5499] [INSPIRE]. 
[71] P. de Aquino, F. Maltoni, K. Mawatari and B. Oexl, Light gravitino production in association with gluinos at the LHC, JHEP 10 (2012) 008 [arXiv:1206.7098] [INSPIRE].

[72] C. Degrande, C. Duhr, B. Fuks, D. Grellscheid, O. Mattelaer, et al., UFO - The universal FeynRules output, Comput. Phys. Commun. 183 (2012) 1201 [arXiv:1108.2040] [INSPIRE].

[73] N.D. Christensen and C. Duhr, FeynRules - Feynman rules made easy, Comput. Phys. Commun. 180 (2009) 1614 [arXiv:0806.4194] [INSPIRE].

[74] N.D. Christensen, P. de Aquino, C. Degrande, C. Duhr, B. Fuks, et al., A comprehensive approach to new physics simulations, Eur. Phys. J. C 71 (2011) 1541 [arXiv:0906.2474] [INSPIRE].

[75] C. Duhr and B. Fuks, A superspace module for the FeynRules package, Comput. Phys. Commun. 182 (2011) 2404 [arXiv:1102.4191] [INSPIRE].

[76] S. Catani, Y.L. Dokshitzer, M. Seymour and B. Webber, Longitudinally invariant $K_{t}$ clustering algorithms for hadron hadron collisions, Nucl. Phys. B 406 (1993) 187 [InSPIRE].

[77] M. Cacciari and G.P. Salam, Dispelling the $N^{3}$ myth for the $K_{t}$ jet-finder, Phys. Lett. B 641 (2006) 57 [hep-ph/0512210] [INSPIRE].

[78] E. Conte, B. Fuks and G. Serret, MadAnalysis 5, a user-friendly framework for collider phenomenology, arXiv:1206.1599 [INSPIRE]. 